\title{
Estimation of the XUV radiation onto close planets and their evaporation ${ }^{\star}$
}

\author{
J. Sanz-Forcada ${ }^{1}$, G. Micela ${ }^{2}$, I. Ribas ${ }^{3}$, A. M. T. Pollock ${ }^{4}$, C. Eiroa ${ }^{5}$, \\ A. Velasco ${ }^{1,6}$, E. Solano ${ }^{1,6}$, and D. García-Álvarez ${ }^{7,8}$ \\ ${ }^{1}$ Departamento de Astrofísica, Centro de Astrobiología (CSIC-INTA), ESAC Campus, PO Box 78, 28691 Villanueva de la Cañada, \\ Madrid, Spain \\ e-mail: jsanz@cab.inta-csic.es \\ 2 INAF - Osservatorio Astronomico di Palermo G. S. Vaiana, Piazza del Parlamento, 1, 90134, Palermo, Italy \\ ${ }^{3}$ Institut de Ciènces de l'Espai (CSIC-IEEC), Campus UAB, Fac. de Ciències, Torre C5-parell-2a planta, 08193 Bellaterra, Spain \\ ${ }^{4}$ XMM-Newton SOC, European Space Agency, ESAC, Apartado 78, 28691 Villanueva de la Cañada, Madrid, Spain \\ 5 Dpto. de Física Teórica, C-XI, Facultad de Ciencias, Universidad Autónoma de Madrid, Cantoblanco, 28049 Madrid, Spain \\ ${ }^{6}$ Spanish Virtual Observatory, Centro de Astrobiología (CSIC-INTA), ESAC Campus, Madrid, Spain \\ 7 Instituto de Astrofísica de Canarias, 38205 La Laguna, Spain \\ 8 Grantecan CALP, 38712 Breña Baja, La Palma, Spain
}

Received 27 January 2011 / Accepted 1 May 2011

\begin{abstract}
Context. The current distribution of planet mass vs. incident stellar X-ray flux supports the idea that photoevaporation of the atmosphere may take place in close-in planets. Integrated effects have to be accounted for. A proper calculation of the mass loss rate through photoevaporation requires the estimation of the total irradiation from the whole XUV (X-rays and extreme ultraviolet, EUV) range. Aims. The purpose of this paper is to extend the analysis of the photoevaporation in planetary atmospheres from the accessible X-rays to the mostly unobserved EUV range by using the coronal models of stars to calculate the EUV contribution to the stellar spectra. The mass evolution of planets can be traced assuming that thermal losses dominate the mass loss of their atmospheres.

Methods. We determine coronal models for 82 stars with exoplanets that have X-ray observations available. Then a synthetic spectrum is produced for the whole XUV range ( 1-912 $⿱$ ). The determination of the EUV stellar flux, calibrated with real EUV data, allows us to calculate the accumulated effects of the XUV irradiation on the planet atmosphere with time, as well as the mass evolution for planets with known density.

Results. We calibrate for the first time a relation of the EUV luminosity with stellar age valid for late-type stars. In a sample of 109 exoplanets, few planets with masses larger than $\sim 1.5 M_{\mathrm{J}}$ receive high XUV flux, suggesting that intense photoevaporation takes place in a short period of time, as previously found in X-rays. The scenario is also consistent with the observed distribution of planet masses with density. The accumulated effects of photoevaporation over time indicate that HD 209458b may have lost $0.2 M_{\mathrm{J}}$ since an age of 20 Myr.

Conclusions. Coronal radiation produces rapid photoevaporation of the atmospheres of planets close to young late-type stars. More complex models are needed to explain the observations fully. Spectral energy distributions in the XUV range are made available for stars in the sample through the Virtual Observatory for the use in future planet atmospheric models.
\end{abstract}

Key words. planetary systems - stars: coronae - astrobiology - X-rays: stars

\section{Introduction}

After 15 years of exoplanetary science, the discipline has reached a point at which it is possible to study in more detail the physical properties of planets, their formation and evolution. With more than 500 exoplanets known to date, it is possible to explore relations between the planets and their host stars. In particular, the mass of the planets and the atmospheric conditions are partly linked to the stellar radiation. Once the planet is formed and the original disc is dissipated, the main agent interacting with the atmosphere should be the high-energy emission from the corona of the star, for late type stars (Lammer et al. 2003; Erkaev et al. 2007; Penz et al. 2008; Cecchi-Pestellini et al. 2009; Sanz-Forcada et al. 2010b, and references therein). Recently Sanz-Forcada et al. (2010b, hereafter Paper I) noticed

^ Appendices and Tables 3 and 4 are available in electronic form at http://www. aanda.org that erosion in exoplanets may be taking place as an effect of coronal radiation that vaporizes the atmosphere of close-in planets: the authors showed that the distribution of planet masses with the X-ray flux at the planet implies that the least massive planets currently receive a high X-ray flux. This is interpreted as the effect of radiation erosion in the long term.

Photons with $\lambda<912 \AA$ can ionize the hydrogen atoms that are assumed to be the main component of the atmospheres of giant planets. The effects of X-rays $(\lambda \lambda 5-100)$ and extreme ultraviolet (EUV, $\lambda \lambda$ 100-912) photons take place at different heights in the atmosphere of the planet. While EUV photons mainly ionize the atoms in the upper atmosphere, X-rays penetrate deeper into the atmosphere. The free electrons carry a high momentum, producing a cascade of collisions, while the X-rays photons are absorbed in the atmosphere (Cecchi-Pestellini et al. 2009). These collisions heat the atmosphere, which leads to its "inflation" and eventually the evaporation of a part of it. The 
gravity of the planet acts as protection, trying to keep the atmosphere attached to the planet. If we assume that the planet atmosphere is mainly composed of hydrogen, and all the photons in the whole XUV range (X-rays+EUV) are absorbed and contribute to the heating of the atmosphere, it is possible to calculate the mass loss of the atmosphere by balancing the losses with the planet gravity (Watson et al. 1981; Lammer et al. 2003). Mass loss also takes place through the Roche lobe for close-in planets (Erkaev et al. 2007; see also Lecavelier des Etangs et al. 2004; Jaritz et al. 2005, for detailed simulations of the escape through the Roche lobe). The resulting formula (Paper I) is

$\dot{M}=\frac{\pi R_{\mathrm{p}}^{3} F_{\mathrm{XUV}}}{\mathrm{G} K M_{\mathrm{p}}}$

where $K(K \leq 1)$ accounts for the planet radius Roche lobe losses (Erkaev et al. 2007), $F_{\mathrm{XUV}}$ is the X-ray and EUV flux at the planet orbit, and $\mathrm{G}$ is the gravitational constant ${ }^{1}$. Baraffe et al. (2004) consider that evaporation actually takes place at a point somewhere above the planet radius $R_{\mathrm{p}}$, at the "expansion radius" $R_{1}$. However, the bulk of the XUV radiation should be absorbed by material enclosed within the planet radius, so we assume that $R_{1} \simeq R_{\mathrm{p}}$. The formula can be simplified using the mean density of the planet $(\rho)$, and assuming that $K \simeq 1$ (valid for most cases, and a lower limit to the mass loss in any case):

$\dot{M}=\frac{3 F_{\mathrm{XUV}}}{4 \mathrm{G} \rho}$.

Stars in the range of late-F to mid-M stellar types are characterized by coronae with temperatures of $\sim 1 \mathrm{MK}$, exceeding $10 \mathrm{MK}$ in the most active cases. The high-temperature material in the transition region $(\sim \log T=4-5.8)$ and corona $(\sim \log T=$ 5.8-7.4) emits copious X-rays and EUV flux. Fast rotators have hotter coronae, resulting in higher XUV fluxes. Current astronomical instruments give access to the X-rays band only. More energetic flux $(\lambda \lesssim 1 \AA$ ) is negligible even for active stars, while the radiation in the EUV band is severely absorbed by the neutral and molecular hydrogen in the interstellar medium (ISM). There are no missions currently observing in the EUV band, and the few data from past telescopes, such as the EUVE (Extreme UltraViolet Explorer), are limited to the closest stars in the range $\lambda \lambda 100-400$. Only one star hosting an exoplanet has been observed in this band, $\epsilon$ Eri (Sanz-Forcada et al. 2003a).

The present-day distribution of planet masses should reflect the accumulated effects of mass loss in the atmospheres of the planet over time (Paper I). Moreover, if we know the evolution of the emission in the whole XUV band, we should be able to trace the planet evolution, given an accurate knowledge of the density of the planet, according to Eq. (2). Since younger stars have faster rotation, their coronae emit more XUV flux, which therefore decreases with time. The evolution of the X-ray emission with age has been studied for the Sun (e.g. Maggio et al. 1987; Ayres 1997; Ribas et al. 2005) and extended to G and M stars (Penz \& Micela 2008; Penz et al. 2008). A relation using late F to early $\mathrm{M}$ stars has been calibrated by Garcés et al. (in prep.) in the X-ray band, allowing us to calculate the age of the stars from its X-ray emission (Paper I) and to trace the time evolution of this emission. In the EUV band the time evolution of the emission has only been studied for the Sun (Ribas et al. 2005).

\footnotetext{
1 A factor of 4, used in Penz et al. (2008) and Paper I, was removed to account for geometrical considerations, as explained in Eq. (21) of Erkaev et al. (2007)
}

Table 1. Observation log of stars with exoplanets ${ }^{a}$.

\begin{tabular}{|c|c|c|c|c|c|c|c|}
\hline \multirow{2}{*}{$\begin{array}{l}\text { Star name } \\
14 \text { Her }\end{array}$} & \multicolumn{2}{|c|}{$\begin{array}{c}\text { Measured coordinates } \\
\alpha, \delta(\mathrm{J} 2000.0)\end{array}$} & \multirow{2}{*}{$\begin{array}{c}\text { Date } \\
2005 / 09 / 11\end{array}$} & \multirow{2}{*}{$\begin{array}{l}\text { Instr. }^{a} \\
\text { EPIC }\end{array}$} & \multirow{2}{*}{$\begin{array}{c}\begin{array}{c}t \\
(\mathrm{ks})\end{array} \\
5\end{array}$} & \multirow{2}{*}{$\begin{array}{r}S / N \\
4.9\end{array}$} & \multirow[t]{2}{*}{ Notes } \\
\hline & $16: 10: 24.6$ & $+43: 49: 01$ & & & & & \\
\hline 16 Cyg B & $19: 41: 48.9$ & $+50: 31: 28$ & $2008 / 11 / 08$ & EPIC & 11 & 2.0 & \\
\hline $2 \mathrm{M} 1207 \mathrm{~A}$ & $12: 07: 33.5$ & $-39: 32: 54$ & $2003 / 03 / 03$ & ACIS & 50 & 0.4 & \\
\hline \multirow[t]{2}{*}{30 Ari B } & $02: 36: 57.6$ & $+24: 38: 52$ & $2001 / 01 / 16$ & EPIC & 20 & 244 & \\
\hline & & & $2001 / 01 / 17$ & EPIC & 34 & & \\
\hline $47 \mathrm{UMa}$ & $10: 59: 28.4$ & $+40: 25: 46$ & $2006 / 06 / 11$ & EPIC & 8 & 4.8 & \\
\hline $51 \mathrm{Peg}$ & $22: 57: 28.1$ & $+20: 46: 08$ & $2008 / 12 / 06$ & ACIS & 5 & 2.6 & \\
\hline $55 \mathrm{Cnc}$ & $08: 52: 35.7$ & $+28: 19: 47$ & $2009 / 04 / 11$ & EPIC & 12 & 14.0 & \\
\hline$\beta \mathrm{Pic}$ & $05: 47: 17.1$ & $-51: 03: 59$ & $2004 / 01 / 04$ & EPIC & 68 & 5.7 & \\
\hline$\epsilon$ Eri & $03: 32: 55.9$ & $-09: 27: 31$ & $2003 / 01 / 19$ & EPIC & 12 & 297 & \\
\hline GJ 86 & $02: 10: 28.1$ & $-50: 49: 19$ & $2008 / 06 / 10$ & EPIC & 15 & 43.0 & WD in field \\
\hline GJ 317 & $08: 40: 59.0$ & $-23: 27: 15$ & $2009 / 04 / 20$ & EPIC & 18 & 9.6 & \\
\hline GJ 436 & $11: 42: 11.6$ & $+26: 42: 16$ & $2008 / 12 / 10$ & EPIC & 30 & 14.5 & \\
\hline GJ 674 & $17: 28: 40.3$ & $-46: 53: 50$ & $2008 / 09 / 05$ & EPIC & 44 & 179 & \\
\hline GJ 876 & $22: 53: 17.3$ & $-14: 15: 55$ & $2008 / 11 / 14$ & EPIC & 23 & 34.7 & \\
\hline GQ Lup & $15: 49: 12.1$ & $-35: 39: 05$ & $2008 / 08 / 16$ & EPIC & 8 & 31.7 & \\
\hline HD 4308 & $00: 44: 39.4$ & $-65: 39: 05$ & $2008 / 12 / 02$ & EPIC & 9 & 2.5 & \\
\hline HD 20367 & $03: 17: 40.1$ & $+31: 07: 37$ & $2005 / 02 / 11$ & EPIC & 10 & 140 & \\
\hline HD 27442 & $04: 16: 29.0$ & $-59: 18: 09$ & $2009 / 02 / 10$ & EPIC & 7 & 2.7 & \\
\hline HD 46375 & $06: 33: 12.4$ & $+05: 27: 49$ & $2005 / 10 / 14$ & EPIC & 8 & 7.3 & \\
\hline HD 49674 & $06: 51: 30.9$ & $+40: 52: 03$ & $2006 / 04 / 10$ & EPIC & 8 & 6.5 & \\
\hline HD 50554 & $06: 54: 42.8$ & $+24: 14: 43$ & $2006 / 04 / 16$ & EPIC & 9 & 2.7 & \\
\hline HD 52265 & $07: 00: 18.0$ & $-05: 22: 01$ & $2008 / 09 / 19$ & EPIC & 9 & 5.4 & \\
\hline HD 70642 & $08: 21: 28.2$ & $-39: 42: 18$ & $2006 / 04 / 08$ & EPIC & 13 & 4.2 & \\
\hline HD 75289 & $08: 47: 40.1$ & $-41: 44: 14$ & $2005 / 04 / 28$ & EPIC & 8 & 2.3 & \\
\hline HD 93083 & $10: 44: 20.9$ & $-33: 34: 38$ & $2008 / 05 / 26$ & EPIC & 12 & 6.3 & \\
\hline HD 95089 & $10: 58: 47.7$ & $+01: 43: 44$ & $2009 / 05 / 26$ & EPIC & 37 & 0.8 & \\
\hline HD 99492 & $11: 26: 45.9$ & $+03: 00: 24$ & $2008 / 06 / 19$ & EPIC & 24 & 11.3 & \\
\hline HD 101930 & $11: 43: 30.1$ & $-58: 00: 21$ & $2009 / 01 / 06$ & EPIC & 2 & 0.4 & \\
\hline HD 102195 & $11: 45: 42.2$ & $+02: 49: 16$ & $2008 / 06 / 15$ & EPIC & 18 & 53.1 & \\
\hline HD 108147 & $12: 25: 46.2$ & $-64: 01: 20$ & $2002 / 08 / 10$ & EPIC & 6 & 4.2 & \\
\hline HD 111232 & $12: 48: 51.8$ & $-68: 25: 29$ & $2008 / 07 / 29$ & EPIC & 9 & 0.9 & \\
\hline HD 114386 & $13: 10: 39.7$ & $-35: 03: 20$ & $2008 / 07 / 29$ & EPIC & 9 & 3.0 & \\
\hline HD 114762 & $13: 12: 19.7$ & $+17: 31: 02$ & $2004 / 06 / 28$ & EPIC & 29 & 1.4 & $\mathrm{dM}$ in field \\
\hline HD 114783 & $13: 12: 43.7$ & $-02: 15: 54$ & $2009 / 01 / 22$ & EPIC & 8 & 3.0 & \\
\hline HD 130322 & $14: 47: 32.8$ & $-00: 16: 54$ & $2005 / 07 / 21$ & EPIC & 7 & 7.7 & \\
\hline HD 154345 & $17: 02: 36.5$ & $+47: 05: 02$ & $2008 / 12 / 25$ & EPIC & 8 & 9.0 & \\
\hline HD 164922 & $18: 02: 33.4$ & $+26: 18: 43$ & $2009 / 03 / 19$ & EPIC & 9 & 1.0 & \\
\hline \multirow[t]{5}{*}{ HD 179949} & $19: 15: 33.3$ & $-24: 10: 46$ & $2005 / 05 / 21$ & ACIS & 30 & 101 & \\
\hline & & & $2005 / 05 / 22$ & ACIS & 30 & & \\
\hline & & & $2005 / 05 / 29$ & ACIS & 30 & & \\
\hline & & & $2005 / 05 / 30$ & ACIS & 32 & & \\
\hline & & & $2005 / 05 / 31$ & ACIS & 30 & & \\
\hline HD 187123 & $19: 46: 57.9$ & $+34: 25: 09$ & $2006 / 04 / 21$ & EPIC & 16 & 1.4 & \\
\hline HD 189733 & 20:00:43.8 & $+22: 42: 34$ & $2007 / 04 / 17$ & EPIC & 43 & 92.5 & \\
\hline HD 190360 & $20: 03: 37.9$ & $+29: 53: 45$ & $2005 / 04 / 25$ & EPIC & 4 & 1.4 & \\
\hline HD 195019 & $20: 28: 18.6$ & $+18: 46: 10$ & $2006 / 04 / 24$ & EPIC & 10 & 2.7 & $\mathrm{dK}$ in field \\
\hline HD 209458 & $22: 03: 10.8$ & $+18: 53: 03$ & $2006 / 11 / 15$ & EPIC & 31 & 1.8 & \\
\hline HD 216435 & $22: 53: 38.1$ & $-48: 35: 55$ & $2006 / 04 / 21$ & EPIC & 7 & 11.9 & \\
\hline HD 216437 & $22: 54: 39.6$ & $-70: 04: 26$ & $2005 / 04 / 13$ & EPIC & 6 & 4.0 & \\
\hline HD 217107 & $22: 58: 15.7$ & $-02: 23: 43$ & $2005 / 05 / 16$ & EPIC & 7 & 2.3 & \\
\hline \multirow[t]{2}{*}{ HD 218566} & 23:09:10.9 & $-02: 15: 39$ & $2001 / 06 / 10$ & EPIC & 3 & 6.8 & \\
\hline & & & $2004 / 06 / 05$ & EPIC & 10 & & \\
\hline HD 330075 & $15: 49: 37.7$ & $-49: 57: 48$ & $2005 / 08 / 07$ & EPIC & 16 & 3.1 & \\
\hline HR 8799 & $23: 07: 28.8$ & $+21: 08: 02$ & $2009 / 08 / 30$ & ACIS & 10 & 6.5 & \\
\hline \multirow[t]{2}{*}{$\mu$ Ara } & $17: 44: 08.7$ & $-51: 50: 04$ & $2008 / 09 / 06$ & EPIC & 6 & 2.9 & \\
\hline & & & $2008 / 10 / 02$ & EPIC & 9 & & \\
\hline NGC 24233 & $07: 37: 09.2$ & $-13: 54: 24$ & $2008 / 05 / 05$ & EPIC & 9 & 1.1 & \\
\hline Pollux & $07: 45: 18.8$ & $+28: 01: 33$ & $2001 / 04 / 26$ & EPIC & 32 & 34.0 & \\
\hline$\tau$ Boo & $13: 47: 15.9$ & $+17: 27: 22$ & $2003 / 06 / 24$ & EPIC & 56 & 317 & $\mathrm{dM} 2$ in field \\
\hline \multirow[t]{4}{*}{$v$ And } & $01: 36: 47.7$ & $+41: 24: 15$ & $2009 / 10 / 20$ & ACIS & 15 & 52.0 & \\
\hline & & & $2009 / 10 / 22$ & ACIS & 15 & & \\
\hline & & & $2009 / 10 / 27$ & ACIS & 14 & & \\
\hline & & & $2009 / 10 / 29$ & ACIS & 14 & & \\
\hline
\end{tabular}

Notes. ${ }^{(a)}$ XMM-Newton (EPIC) or Chandra (ACIS) instrument used to measure the X-ray flux.

In this work we extend the analysis of Paper I to the EUV band to account for all stellar radiation capable to ionize hydrogen in a planet atmosphere. Lecavelier Des Etangs (2007) extrapolated the EUV flux from the X-ray flux, using the Sun 
Table 2. ROSAT/PSPC X-ray flux $(0.12-2.48 \mathrm{keV})$ of stars with exoplanets.

\begin{tabular}{lccccl}
\hline \hline Star name & \multicolumn{2}{c}{$\begin{array}{c}\text { Coordinates } \\
\alpha, \delta(J 2000.0)\end{array}$} & $S / N$ & $\begin{array}{c}\log f_{\mathrm{X}} \\
\left(\mathrm{erg} \mathrm{s}^{-1} \mathrm{~cm}^{-2}\right)\end{array}$ & Notes \\
\hline 18 Del & $20: 58: 25.9$ & $+10: 50: 21$ & 4.5 & -12.48 & \\
1RXS 1609 & $16: 09: 30.3$ & $-21: 04: 58$ & 3.5 & -12.52 & K-M in field \\
4 UMa & $08: 40: 12.8$ & $+64: 19: 40$ & 11.6 & -13.26 & \\
61 Vir & $13: 18: 24.3$ & $-18: 18: 40$ & 5.7 & -13.06 & \\
$\alpha$ Ari & $02: 07: 10.4$ & $+23: 27: 44$ & 5.1 & -13.50 & \\
BD-10 3166 & $10: 58: 28.8$ & $-10: 46: 13$ & 3.1 & $<-12.52$ & +dM5, uncertain $d$ \\
$\gamma$ Cep & $23: 39: 20.8$ & $+77: 37: 56$ & 8.1 & $<-13.03$ & dM4 in field \\
GJ 176 & $04: 42: 55.8$ & $+18: 57: 29$ & 3.2 & -12.62 & \\
GJ 832 & $21: 33: 34.0$ & $-49: 00: 32$ & 7.3 & -12.69 & \\
GJ 3021 & $00: 16: 12.7$ & $-79: 51: 04$ & 7.8 & $<-11.62$ & dM4 in field \\
HD 3651 & $00: 39: 21.8$ & $+21: 15: 01$ & 4.5 & -12.89 & \\
HD 10647 & $01: 42: 29.3$ & $-53: 44: 27$ & N/A & -12.24 & \\
HD 38529 & $05: 46: 34.9$ & $+01: 10: 05$ & 5.3 & -12.37 & dM2 in field \\
HD 41004 A & $05: 59: 49.6$ & $-48: 14: 22$ & 7.5 & $<-12.02$ & \\
HD 48265 & $06: 40: 01.7$ & $-48: 32: 31$ & 6.0 & -12.43 & \\
HD 70573 & $08: 22: 50.0$ & $+01: 51: 33$ & 4.0 & -12.31 & uncertain $d$ \\
HD 87883 & $10: 08: 43.1$ & $+34: 14: 32$ & N/A & -12.99 & \\
HD 89744 & $10: 22: 10.6$ & $+41: 13: 46$ & 7.6 & -13.17 & \\
HD 102365 & $11: 46: 31.1$ & $-40: 30: 01$ & 4.8 & -13.20 & faint dM4 in field \\
HD 128311 & $14: 36: 00.6$ & $+09: 44: 47$ & 7.5 & -12.04 & \\
HD 142415 & $15: 57: 40.8$ & $-60: 12: 00$ & 5.0 & -12.50 & \\
HD 147513 & $16: 24: 01.3$ & $-39: 11: 34$ & 16.2 & -11.40 & \\
HD 150706 & $16: 31: 17.6$ & $+79: 47: 23$ & 12.0 & -12.13 & \\
HD 169830 & $18: 27: 49.5$ & $-29: 49: 00$ & 16.8 & -12.94 & F9V in field \\
HD 176051 & $18: 57: 01.5$ & $+32: 54: 06$ & 7.2 & $<-12.27$ & \\
HIP 75458 & $15: 24: 55.8$ & $+58: 57: 57$ & 3.5 & -13.66 & ROSAT/WGA detector \\
HIP 79431 & $16: 12: 41.8$ & $-18: 52: 31$ & 3.6 & -13.50 & \\
HR 810 & $02: 42: 33.5$ & $-50: 48: 01$ & 7.0 & -11.67 & \\
\hline
\end{tabular}

Notes. The star GJ 667C (M1V) was well detected with ROSAT/PSPC $(S / N=7.1)$, but its $\mathrm{X}$-ray emission is attributed to its companions GJ 667A (K3V) and GJ 667B (K5V), at 43".

as pattern for all kind of stars, without checking whether the relation is valid at all levels of activity, and therefore ages. Since there are essentially no measurements in the range $\sim 400-912 \AA$ for stars other than the Sun, we have used coronal models to synthesize the spectral energy distribution (SED) in the whole EUV range, and tested the results in X-rays and the lower wavelengths of the band (100-400 $⿱$ ) for a few cases with EUV spectra available. We have set up a database (http://sdc.cab. inta-csic.es/xexoplanets) that is freely available (within the Spanish Virtual Observatory), "X-exoplanets". The database includes synthetic SEDs in the range 1-1200 ̊ for all stars listed in Table 1. Objects will be incorporated in the future as they are observed in X-rays.

The paper is structured as follows: Sect. 2 describes the observations, Sect. 3 extends the X-ray analysis to the EUV band; Sect. 4 shows the results found for the sample, which are discussed in Sect. 5, and we list the conclusions of the work in Sect. 6.

\section{Observations}

We used data acquired with the X-ray telescopes XMM-Newton, Chandra and ROSAT (Tables 1,2). XMM-Newton and Chandra data were taken from their public archives, including data awarded to us as P.I. or co-I. (XMM prop. ID \#020653, \#020000, \#055102). Data were reduced following standard procedures, removing time intervals affected by high background, likely produced by space weather events. The expected position of the targets were calculated using the coordinates and proper motions provided by SIMBAD. XMM-Newton/EPIC and Chandra/ACIS have spatial resolution of $6^{\prime \prime}$ and $2^{\prime \prime}$ respectively. The cleaned observations (Table 1) were used to extract the low-resolution spectra provided by XMM-Newton/EPIC and Chandra/ACIS $(E / \Delta E=20-50)$. The ISIS package (Houck \& Denicola 2000) and the Astrophysics Plasma Emission Database (APED, Smith et al. 2001) were used to fit the spectra with coronal models of one to three temperature components (Table 3), depending on the quality of the spectra, and variable stellar coronal abundances, using the generally low value of the ISM absorption. The background spectrum was fitted simultaneously to the source to account for its contribution to the total spectrum. Spectra and light curves for each target are available online (http://sdc.cab. inta-csic.es/xexoplanets) in the "X-exoplanets" database (Sanz-Forcada et al. 2010a), described in detail in Appendix C. Spectra in the XUV range can be used for planet atmospheric models.

The spectra with lowest statistics have a deficient fit. This yields low abundance and very high emission measure, despite of the low temperature observed (emission measures in stellar coronae increase with the their coronal temperature). The use of solar abundance provides a similar fit, but with more realistic values of the emission measure. In these cases we fixed temperature and abundance to the solar values. We use $\log T[\mathrm{~K}]=6.3$ and the solar photospheric abundances of Asplund et al. (2005), corresponding to $[\mathrm{Fe} / \mathrm{H}]=-0.2$ in the scale of Anders \& Grevesse (1989) used in Table 3. The actual model used in the fit has little influence on the calculation of the X-ray $(0.12-2.48 \mathrm{keV}$ or $\sim 5-100 \AA)$ flux displayed in Table 3, but it is important for the extension to the EUV range, as explained in Sect. 3. Table 3 also lists the errors for objects with net count rates with $S / N>3$, and considers the rest of detections as upper limits. We marked GJ 86 as an upper limit to account for the contribution of an unresolved companion.

ROSAT/PSPC observations were added to the sample. We considered only detections with $S / N>3$, given the lower spatial resolution of this instrument $\left(25^{\prime \prime}\right)$, marking as upper limits objects with suspected X-ray bright companions, as indicated in Table 2 (as a reference, a dM3 star may have up to $\left.\log L_{X} \sim 27.5\right)$. To calculate the X-ray flux of the targets, we considered the count rate $(C R)$ reported in HEASARC (http://heasarc.gsfc.nasa.gov/), corresponding to the spectral range $0.12-2.48 \mathrm{keV}$, and then transformed it into flux using $f_{\mathrm{X}}=C R \times 6.19 \times 10^{-12} \mathrm{erg} \mathrm{cm}^{-2} \mathrm{cts}^{-1}$, as proposed by Schmitt et al. (1995) and Huensch et al. (1998), using a hardness ratio of -0.4 (corresponding to a middle activity level star, $\epsilon$ Eri).

The stellar distance, which was not available for three ROSAT stars, was calculated using the spectroscopic parallax method, i.e., comparing the visual magnitude $V$ with the absolute magnitude $M_{\mathrm{V}}$ that corresponds to the spectral type of the star (Cox 2000). We calculated the bolometric luminosity $\left(L_{\mathrm{bol}}\right)$ of each star using the bolometric corrections by Flower (1996) and $B-V$ colors based on spectral types in Cox (2000) if no direct measurements were available in SIMBAD. The calculation of the age of the stars partly depends on $L_{\text {bol }}$ (Paper I). Some X-ray fluxes differ from other surveys (Kashyap et al. 2008; Poppenhaeger et al. 2010) mainly because they calculated the $\mathrm{X}$-ray flux as a count rate conversion for most objects, or because of misidentifications of the stellar position in the field, as described in Paper I.

\section{Extension to the EUV}

In Paper I we calculated the X-ray fluxes that were received at the planet orbit $\left(F_{\mathrm{X}}\right)$. We need to consider the whole flux emitted by the star in the X-ray and EUV range to calculate the mass loss rate of the planet according to Eq. (2). Lecavelier Des Etangs (2007) extrapolated the EUV flux from the X-ray-to-EUV ratio observed in the Sun, assuming the same ratio for all spectral 


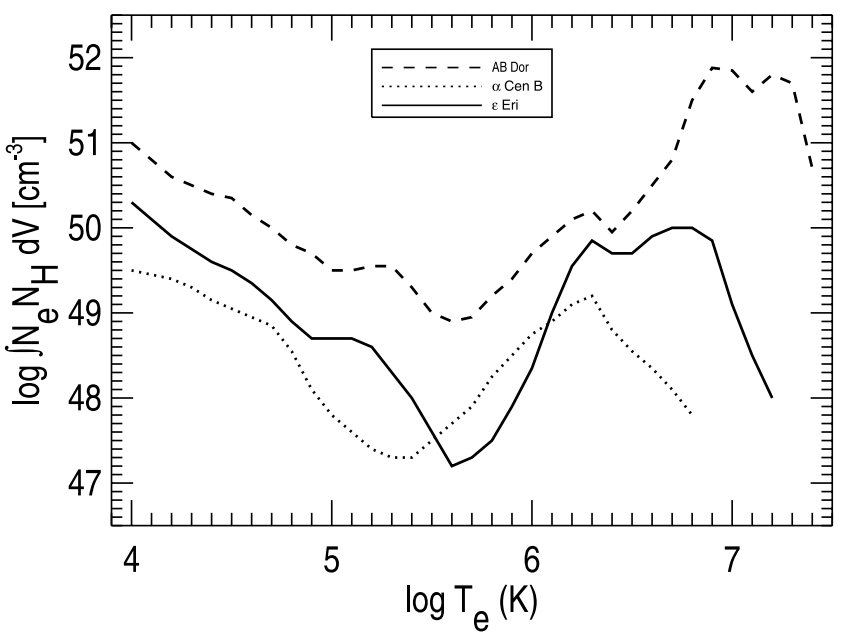

Fig. 1. Emission measure distribution (EMD) of three $\mathrm{K} 2 \mathrm{~V}$ stars with different levels of activity. The shape of solar EMD (Orlando et al. 2001) is similar to that of $\alpha$ Cen B.

types and activity levels (thus age). The few observations of latetype stars in the EUV contradict this view (e.g. Sanz-Forcada et al. 2003a). A better way to determine the radiation in the EUV range is to calculate a synthetic spectrum of each star using a coronal model. Such a model must describe accurately how the mass is distributed with temperature in the corona and transition region, the so-called emission measure distribution (EMD). The EMD is complemented with the abundances of the different elements in the corona. If combined with an appropriate atomic model, it will be possible to predict the spectral energy distribution generated at coronal temperatures. Atomic models in this range have been tested only with solar data, and at the shorter wavelengths $(\lambda \lambda \sim 100-400 \AA)$ with more active stars. We expect that a few lines formed at high temperature have inaccurate calculations or are missing from the models. But the bulk of the emission is already included in the models, and the flux in a given range should be quite accurate. We use in our case the atomic model APED (Smith et al. 2001).

The atomic model needs to be folded with a coronal model. Figure 1 shows the EMDs of three K2V stars with good highresolution spectra that allow us to construct an EMD with a resolution of 0.1 dex in temperature, following the method described in Sanz-Forcada et al. (2003b, and references therein). In general we do not have an accurate determination of the EMD for the stars in the sample, most of them limited to fits with less than three temperature components. Moreover, the observations in X-rays give us access only to the EM at $T \gtrsim 1 \mathrm{MK}$, because lower temperature lines are rare in X-rays. However, we cannot ignore the lower temperature part of the EMD, which corresponds to the transition region, because many lines in the EUV band are formed at $T \lesssim 1 \mathrm{MK}$, notibly He II $\lambda 304 \AA$, one of the strongest lines in the XUV spectrum of most cool stars.

\subsection{Transition region EMD}

It is possible to calculate the EMD at $T \lesssim 1 \mathrm{MK}$ by using lines in the UV (e.g. Dupree et al. 1993), but most of our objects have no UV spectra available. The large sample of EMDs reported by Sanz-Forcada et al. (2003a) showed that the "cool" side of the EMD is approximately proportional to the EMD at $\log T(\mathrm{~K}) \sim 6-6.3$. We checked that a reasonable proportionality exists among the values at these two temperature

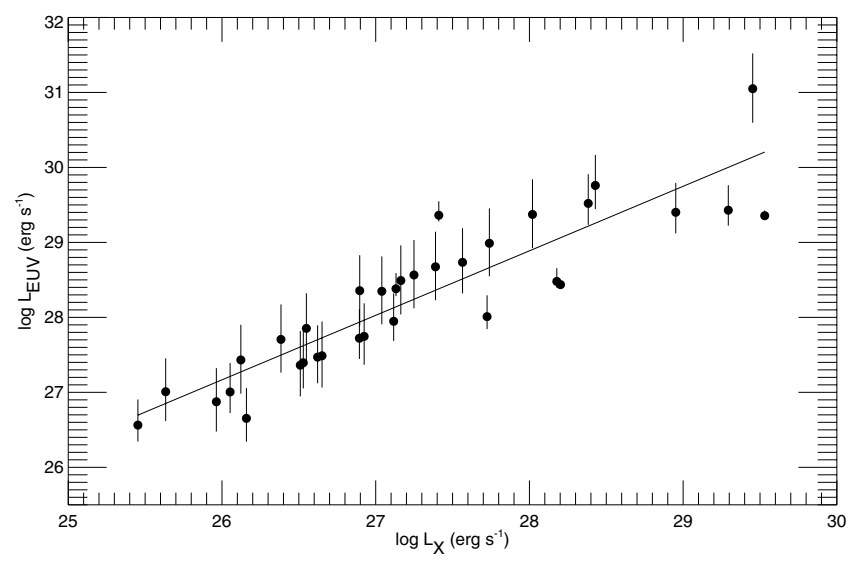

Fig. 2. X-ray vs. EUV luminosity in selected stars of the sample (see text). Uncertainties come from calculating the transition region EMD.

ranges (Appendix A). We calibrated the relation using stars with known EMD at all temperatures, and extrapolated the lower temperature EMD for the stars unobserved in UV with this relation, as described in Appendix A. In particular we chose the EMD of the sample of stars in Sanz-Forcada et al. (2003a) that have well calculated EMD: we separated the sample into three groups depending on the level of activity (interpreted from the amount of EMD found at the highest temperatures). We then defined three parameters: a parameter to account for the transformation between an EMD with 0.1 dex of temperature resolution and a $3 \mathrm{~T}$ fit, the difference between the minimum of the EMD and the local maximum found at $\log T(\mathrm{~K}) \sim 6.2-6.4$, and the slope of the EMD at temperatures below the EMD minimum. We then tested this extrapolation with one star of each of the three classes: AB Dor (Sanz-Forcada et al. 2003b), $\epsilon$ Eri (Sanz-Forcada et al. 2004), and $\alpha$ Cen B (Sect. B), all of type K2V (Fig. 1). The XUV flux calculated using a 3-T model $(\log T(\mathrm{~K}) \geq 5.8)$ and a synthetic EMD $(\log T(\mathrm{~K})<5.8)$ agrees in all cases within 0.37 dex with that calculated using a complete EMD. Therefore, we are confident that the method can be safely applied to the stars in our sample. As a comparison, the solar activity cycle spans $1.7 \mathrm{dex}$ in X-ray luminosity (Orlando et al. 2001). We also used these stars to check how much flux we would be missing if we considered the EMD only above $1 \mathrm{MK}$, for the different levels of activity: we would miss a $3 \%$ of the flux (AB Dor, very active), $19 \%$ (intermediate activity $\epsilon$ Eri), and $40 \%$ ( $\alpha$ Cen B, low activity). We conclude that the extension of the EMD to the cooler temperatures is necessary, especially for the less active stars. The reason is because in $\alpha$ Cen B $91 \%$ of the XUV flux is generated in the EUV band, while for AB Dor only $23 \%$ is in the EUV. Table 4 includes the predicted flux in different bands of the EUV range, with a formal precision of 0.01 dex well below the expected cyclic variability of the stars.

The application of this calculation to the ROSAT data is not possible because we do not have a coronal model for these objects. Instead we calculated a direct relation between X-ray and EUV luminosities for all the XMM and Chandra objects in our sample that have an X-ray flux available (i.e., it is not an upper limit), with the EUV flux calculated as explained. A fit to these data (Fig. 2) yields

$\log L_{\mathrm{EUV}}=(4.80 \pm 1.99)+(0.860 \pm 0.073) \log L_{\mathrm{X}}$

where $L_{\mathrm{X}}$ and $L_{\mathrm{EUV}}$ are the X-ray $(\lambda \lambda 5-100 \AA)$ and EUV ( $\lambda \lambda$ 100-920 $\AA$ ) luminosities, in erg $\mathrm{s}^{-1}$. We then applied this 
Table 5. ROSAT X-ray (5-100 ̊̊) and EUV (100-920 ̊̊) luminosity of stars with exoplanets ${ }^{a}$ (ROSAT). XUV includes the 5-920 Å range.

\begin{tabular}{|c|c|c|c|c|c|c|c|c|c|c|c|c|c|c|}
\hline Planet name & $\begin{array}{l}\text { Sp. type } \\
\text { (star) }\end{array}$ & $\begin{array}{l}\text { Stellar distance } \\
(\mathrm{pc})\end{array}$ & $\begin{array}{c}\log L_{\mathrm{X}} \\
\left(\mathrm{erg} \mathrm{s}^{-1}\right)\end{array}$ & $\begin{array}{l}\log L_{\mathrm{EUV}} \\
\left(\mathrm{erg} \mathrm{s}^{-1}\right)\end{array}$ & $\begin{array}{l}\log L_{\text {bol }} \\
\left(\mathrm{erg} \mathrm{s}^{-1}\right)\end{array}$ & $\begin{array}{l}\text { age } \\
\text { (Gyr) }\end{array}$ & $\begin{array}{c}M_{\mathrm{p}} \sin i \\
\left(m_{\mathrm{J}}\right)\end{array}$ & $\begin{array}{l}a_{\mathrm{p}} \\
(\mathrm{au})\end{array}$ & $\begin{array}{r}\log F_{\mathrm{X}} \\
\quad\left(\mathrm{erg} \mathrm{s}^{-}\right.\end{array}$ & $\begin{array}{l}\log F_{\mathrm{XUV}} \\
\left.-1 \mathrm{~cm}^{-2}\right)\end{array}$ & $\log F_{\mathrm{X}}$ accu & $F_{\text {Xuv accum. }}$ & $\begin{array}{l}\rho \dot{M}_{\mathrm{X}} \\
\left(\mathrm{g}^{2} \mathrm{~s}^{-1}\right.\end{array}$ & $\begin{array}{l}\rho \dot{M}_{\mathrm{XUV}} \\
\left.\mathrm{cm}^{-3}\right)^{b}\end{array}$ \\
\hline 18 Del b & G6III & $73.10 \pm 3.74$ & 29.33 & 30.02 & 35.11 & $\ldots$ & 10.30 & 2.60 & 1.05 & 1.82 & .. & . & $1.3 \mathrm{e}+08$ & $7.5 e+08$ \\
\hline $1 \mathrm{RXS} 1609 \mathrm{~b}$ & $\mathrm{~K} 7 \mathrm{~V}$ & 145 : & 29.88 & 30.50 & 33.20 & 0.09 & 8.00 & 330.00 & -2.61 & -1.90 & 12.91 & 13.72 & $2.8 \mathrm{e}+04$ & $1.4 \mathrm{e}+05$ \\
\hline $4 \mathrm{UMa}$ b & K1III & $77.40 \pm 4.25$ & 28.41 & 29.23 & 35.65 & $\ldots$ & 7.10 & 0.87 & 1.08 & 1.97 & $\ldots$ & $\ldots$ & $1.4 \mathrm{e}+08$ & $1.0 \mathrm{e}+09$ \\
\hline 61 Vir b & G5V & $8.53 \pm 0.05$ & 26.88 & 27.92 & 33.49 & 7.96 & 0.02 & 0.05 & 2.03 & 3.10 & 21.02 & 21.76 & $1.2 \mathrm{e}+09$ & $1.4 \mathrm{e}+10$ \\
\hline 61 Vir c & & & & & & & 0.06 & 0.22 & 0.76 & 1.83 & 19.75 & 21.76 & $6.4 \mathrm{e}+07$ & $7.6 \mathrm{e}+08$ \\
\hline 61 Vir d & & & & & & & 0.07 & 0.48 & 0.08 & 1.15 & 19.07 & 21.76 & $1.3 \mathrm{e}+07$ & $1.6 \mathrm{e}+08$ \\
\hline$\alpha$ Ari b & K2III & $20.21 \pm 0.40$ & 27.19 & 28.18 & 35.51 & $\ldots$ & 1.80 & 1.20 & -0.42 & 0.62 & $\ldots$ & $\ldots$ & $4.3 e+06$ & $4.7 \mathrm{e}+07$ \\
\hline BD-10 $3166 b$ & G4V & $66:$ & $<29.20$ & $<29.91$ & 33.19 & 0.25 & 0.48 & 0.05 & $<4.43$ & $<5.21$ & 20.84 & 21.61 & $(3.0 \mathrm{e}+11)$ & $(1.8 \mathrm{e}+12)$ \\
\hline$\gamma$ Cep b & $\mathrm{K} 2 \mathrm{IV}$ & $13.79 \pm 0.10$ & $<27.33$ & $<28.30$ & 34.63 & 4.08: & 1.60 & 2.04 & $<-0.74$ & $<0.28$ & 18.16: & 18.62: & $(2.0 \mathrm{e}+06)$ & $(2.1 \mathrm{e}+07)$ \\
\hline GJ 176 b & $\mathrm{M} 2.5 \mathrm{~V}$ & $9.42 \pm 0.22$ & 27.41 & 28.37 & 31.74 & 3.62 & 0.03 & 0.07 & 2.32 & 3.33 & 20.12 & 21.43 & $2.4 \mathrm{e}+09$ & $2.4 \mathrm{e}+10$ \\
\hline GJ 832 b & M1.5V & $4.94 \pm 0.03$ & 26.78 & 27.83 & 31.81 & 9.24 & 0.64 & 3.40 & -1.73 & -0.64 & 16.77 & 18.05 & $2.1 \mathrm{e}+05$ & $2.5 \mathrm{e}+06$ \\
\hline GJ 3021 b & G6V & $17.62 \pm 0.16$ & $<28.95$ & $<29.70$ & 33.39 & 0.37 & 3.37 & 0.49 & $<2.12$ & $<2.94$ & 18.91 & 19.61 & $(1.5 e+09)$ & $(9.8 \mathrm{e}+09)$ \\
\hline HD $3651 \mathrm{~b}$ & $\mathrm{~K} 0 \mathrm{~V}$ & $11.11 \pm 0.09$ & 27.27 & 28.25 & 33.36 & 4.46 & 0.20 & 0.28 & 0.91 & 1.94 & 19.47 & 20.23 & $9.2 \mathrm{e}+07$ & $9.8 \mathrm{e}+08$ \\
\hline HD $10647 \mathrm{~b}$ & F8V & $17.35 \pm 0.19$ & 28.31 & 29.15 & 33.75 & 0.95 & 0.93 & 2.03 & 0.25 & 1.14 & 17.83 & 18.47 & $2.0 \mathrm{e}+07$ & $1.6 \mathrm{e}+08$ \\
\hline HD $38529 b$ & G4IV & $42.43 \pm 1.66$ & 28.96 & 29.71 & 34.41 & $0.36:$ & 0.78 & 0.13 & 3.28 & 4.09 & 20.42: & 20.86: & $2.1 \mathrm{e}+10$ & $1.4 \mathrm{e}+11$ \\
\hline HD $39091 \mathrm{~b}$ & G1IV & $18.21 \pm 0.15$ & 27.49 & 28.44 & 33.74 & $3.22:$ & 10.30 & 3.28 & -0.99 & 0.01 & 17.44: & 18.12: & $1.1 \mathrm{e}+06$ & $1.1 \mathrm{e}+07$ \\
\hline HD $41004 \mathrm{~A} \mathrm{~b}$ & $\mathrm{~K} 1 \mathrm{~V}$ & $43.03 \pm 1.89$ & $<29.31$ & $<30.01$ & 33.36 & 0.22 & 2.54 & 1.64 & $<1.43$ & $<2.21$ & 17.79 & 18.50 & $(3.0 \mathrm{e}+08)$ & $(1.8 \mathrm{e}+09)$ \\
\hline HD $48265 \mathrm{~b}$ & G5V & $87.41 \pm 5.50$ & 29.53 & 30.20 & 34.18 & 0.16 & 1.16 & 1.51 & 1.72 & 2.47 & 18.15 & 18.61 & $5.9 \mathrm{e}+08$ & $3.3 e+09$ \\
\hline HD 70573 b & G1-1.5V & $45.7:$ & 29.09 & 29.82 & 33.33 & 0.30 & 6.10 & 1.76 & 1.15 & 1.95 & 17.76 & 18.48 & $1.6 \mathrm{e}+08$ & $1.0 \mathrm{e}+09$ \\
\hline HD 87883 b & $\mathrm{K} 0 \mathrm{~V}$ & $18.06 \pm 0.31$ & 27.60 & 28.54 & 33.10 & 2.73 & 12.10 & 3.60 & -0.96 & 0.02 & 17.17 & 18.00 & $1.2 \mathrm{e}+06$ & $1.2 \mathrm{e}+07$ \\
\hline HD 89744 b & F7V & $38.99 \pm 1.06$ & 28.11 & 28.97 & 34.38 & 1.28 & 7.20 & 0.88 & 0.77 & 1.69 & 18.79 & 19.28 & $6.6 e+07$ & $5.5 \mathrm{e}+08$ \\
\hline HD $102365 \mathrm{~b}$ & $\mathrm{G} 2 \mathrm{~V}$ & $9.24 \pm 0.06$ & 26.81 & 27.86 & 33.49 & 8.83 & 0.05 & 0.46 & 0.04 & 1.12 & 19.10 & 19.84 & $1.2 \mathrm{e}+07$ & $1.5 \mathrm{e}+08$ \\
\hline HD $128311 b$ & $\mathrm{KOV}$ & $16.57 \pm 0.27$ & 28.48 & 29.29 & 33.06 & 0.74 & 2.18 & 1.10 & 0.95 & 1.82 & 18.13 & 18.95 & $1.0 \mathrm{e}+08$ & $7.5 \mathrm{e}+08$ \\
\hline HD $128311 \mathrm{c}$ & & & & & & & 3.21 & 1.76 & 0.54 & 1.41 & 17.72 & 18.95 & $3.9 \mathrm{e}+07$ & $2.9 \mathrm{e}+08$ \\
\hline HD $142415 b$ & G1V & $34.57 \pm 1.00$ & 28.65 & 29.44 & 33.63 & 0.57 & 1.62 & 1.05 & 1.16 & 2.01 & 18.35 & 19.01 & $1.6 \mathrm{e}+08$ & $1.2 \mathrm{e}+09$ \\
\hline HD $147513 b$ & G3/G5V & $12.87 \pm 0.14$ & 28.90 & 29.65 & 33.56 & 0.40 & 1.21 & 1.32 & 1.21 & 2.03 & 18.11 & 18.77 & $1.8 \mathrm{e}+08$ & $1.2 \mathrm{e}+09$ \\
\hline HD $150706 \mathrm{~b}$ & G0 & $27.23 \pm 0.42$ & 28.82 & 29.59 & 33.55 & 0.45 & 1.00 & 0.82 & 1.54 & 2.38 & 18.53 & 19.20 & $3.9 \mathrm{e}+08$ & $2.7 e+09$ \\
\hline HD $169830 \mathrm{~b}$ & F8V & $36.32 \pm 1.20$ & 28.26 & 29.10 & 34.23 & 1.02 & 2.88 & 0.81 & 0.99 & 1.90 & 18.80 & 19.32 & $1.1 \mathrm{e}+08$ & $8.8 \mathrm{e}+08$ \\
\hline HD $169830 \mathrm{c}$ & & & & & & & 4.04 & 3.60 & -0.30 & 0.60 & 17.50 & 19.32 & $5.6 \mathrm{e}+06$ & $4.5 \mathrm{e}+07$ \\
\hline HD $176051 \mathrm{~b}$ & K1V & $14.98 \pm 0.12$ & $<28.22$ & $<29.07$ & 32.86 & 1.09 & 1.50 & 1.76 & $<0.28$ & $<1.19$ & 17.67 & 18.56 & $(2.1 \mathrm{e}+07)$ & $(1.7 \mathrm{e}+08)$ \\
\hline HIP 75458 b & K2III & $31.33 \pm 0.50$ & 27.41 & 28.37 & 35.38 & $\ldots$ & 8.82 & 1.27 & -0.25 & 0.76 & $\ldots$ & $\ldots$ & $6.3 e+06$ & $6.4 \mathrm{e}+07$ \\
\hline HIP 79431 b & M3V & $14.90 \pm 0.79$ & 26.89 & 27.93 & 60.02 & 7.84 & 2.10 & 0.36 & 0.33 & 1.40 & 28.76 & 28.76 & $2.4 \mathrm{e}+07$ & $2.8 \mathrm{e}+08$ \\
\hline HR $810 \mathrm{~b}$ & G0V & $17.24 \pm 0.16$ & 28.79 & 29.56 & 33.80 & 0.47 & 2.26 & 0.93 & 1.41 & 2.25 & 18.49 & 19.11 & $2.9 \mathrm{e}+08$ & $2.0 \mathrm{e}+09$ \\
\hline
\end{tabular}

Notes. ${ }^{(a)}$ Planet data from The Extrasolar Planets Encyclopedia (http://exoplanet. eu). ${ }^{(b)} 1 M_{\mathrm{J}} \mathrm{Gyr}^{-1}=6.02 \times 10^{13} \mathrm{~g} \mathrm{~s}^{-1}$.

conversion to the ROSAT data. The calculated EUV luminosities are listed in Table 5.

\subsection{EUV luminosity evolution with age}

We calibrated a relation of age- $L_{\text {EUV }}$ to calculate better the effects of the radiation in the whole XUV range. We selected all stars in our sample for the calibration, except for those with only an upper limit of the X-ray flux and stars that show a formal result with age larger than 20 Gyr (those with spectral type A and some $\mathrm{M}$ stars). The age determination is made with the X-ray luminosity as explained in next section. The fit (Fig. 3) follows a power-law relation

$\log L_{\mathrm{EUV}}=(29.12 \pm 0.11)-(1.24 \pm 0.15) \log \tau$

where $\tau$ is the age in Gyr. This relation was used to calculate the accumulated effects of the coronal radiation in the planet atmosphere as listed in Tables 5 and 6. Although Ribas et al. (2005) studied the time evolution of the EUV luminosity, this is the first time that a general relation between age and $L_{\mathrm{EUV}}$ has been calculated. Ribas et al. (2005) studied the spectral energy distributions of six solar analogs covering a wide range $(0.1-6.7 \mathrm{Gyr})$ of stellar ages in the X-rays and UV regimes. The authors used real spectra in the ranges $1-360 \AA$ and $920-1950 \AA$ as available. The distribution of stellar fluxes in the different bands with the stellar age was used to calibrate the time evolution of the high-energy irradiance in these bands. The results were used to interpolate

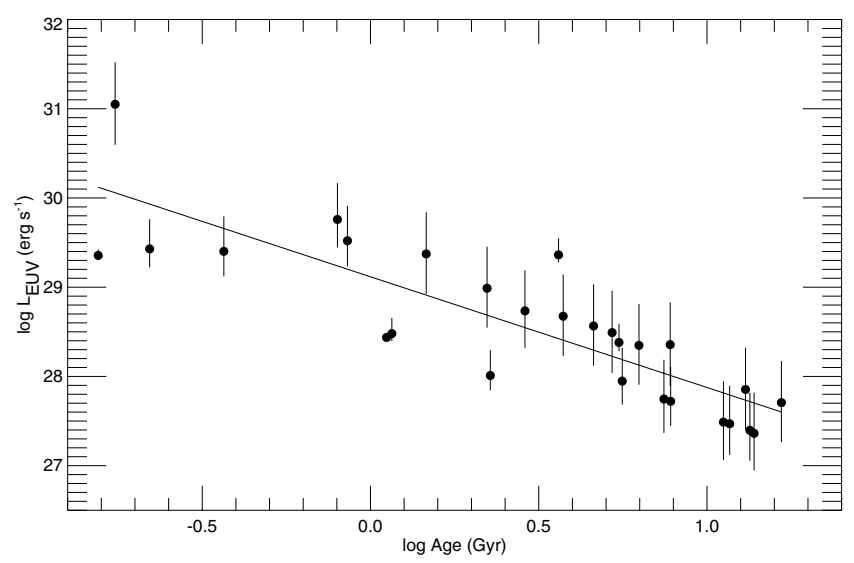

Fig. 3. Distribution of EUV luminosities against age (in Gyr) determined using the X-ray luminosities. The line indicates the best linear fit to the sample.

the evolution in the 360-920 $\mathrm{A}$ band, resulting in a less steeper dependence (exponent $\sim-1.0$ in this range, -1.20 in $100-360 \AA$ ) than in our case $(-1.24$ in $100-920 \AA)$.

\section{Results}

Processes of mass loss in the atmosphere of a planet are not well understood. Once the circumstellar disk is dissipated it is 
A\&A 532, A6 (2011)

Table 6. X-ray (5-100 ̊) and EUV (100-920 Å) luminosity of stars with exoplanets ${ }^{a}$ (XMM-Newton and Chandra).

\begin{tabular}{|c|c|c|c|c|c|c|c|c|c|c|c|c|c|c|}
\hline \multirow{2}{*}{$\begin{array}{l}\text { Planet name } \\
14 \text { Her b }\end{array}$} & \multirow{2}{*}{$\begin{array}{c}\begin{array}{c}\text { Sp. type } \\
\text { (star) }\end{array} \\
\text { K0V }\end{array}$} & \multirow{2}{*}{$\begin{array}{c}\text { Stellar distance } \\
(\mathrm{pc})\end{array}$} & \multirow{2}{*}{$\begin{array}{c}\begin{array}{c}\log L_{\mathrm{X}} \\
\left(\mathrm{erg} \mathrm{s}^{-1}\right)\end{array} \\
26.92\end{array}$} & \multirow{2}{*}{$\begin{array}{c}\log L_{\mathrm{EUV}} \\
\left(\mathrm{erg} \mathrm{s}^{-1}\right)\end{array}$} & \multirow{2}{*}{$\begin{array}{c}\begin{array}{c}\log L_{\mathrm{bol}} \\
\left(\mathrm{erg} \mathrm{s}^{-1}\right)\end{array} \\
33.43\end{array}$} & \multirow{2}{*}{$\begin{array}{c}\begin{array}{c}\text { age } \\
(\mathrm{Gyr})\end{array} \\
7.45\end{array}$} & \multirow{2}{*}{$\begin{array}{c}\begin{array}{c}M_{\mathrm{p}} \sin i \\
\left(m_{\mathrm{J}}\right)\end{array} \\
4.64\end{array}$} & \multirow{2}{*}{$\begin{array}{c}\begin{array}{c}a_{\mathrm{p}} \\
(\mathrm{au})\end{array} \\
2.77\end{array}$} & \multirow{2}{*}{$\begin{array}{c}\begin{array}{c}\log F_{\mathrm{X}} \\
\left(\mathrm{erg} \mathrm{s}^{-1}\right.\end{array} \\
-1.41\end{array}$} & \multirow{2}{*}{$\begin{array}{l}\log F_{\text {XUV }} \\
\left.-\mathrm{cm}^{-2}\right) \\
-0.53\end{array}$} & \multicolumn{2}{|c|}{$\begin{array}{c}\log F_{\mathrm{X}} \text { accum. } \log F_{\text {XUV }} \text { accum. } \\
\left(\mathrm{erg} \mathrm{cm}^{-2}\right)\end{array}$} & \multirow{2}{*}{$\begin{array}{c}\begin{array}{c}\rho \dot{M}_{\mathrm{X}} \\
\left(\mathrm{g}^{2} \mathrm{~s}^{-1}\right.\end{array} \\
4.4 \mathrm{e}+05\end{array}$} & $\begin{array}{l}\rho \dot{M}_{\mathrm{XUV}} \\
\left.\mathrm{cm}^{-3}\right)^{c}\end{array}$ \\
\hline & & & & & & & & & & & 17.52 & 18.28 & & $3.3 e+06$ \\
\hline 16 Cyg B b & $\mathrm{G} 2.5 \mathrm{~V}$ & $21.62 \pm 0.23$ & $<26.71$ & $<28.04$ & 33.78 & 10.31 & 1.68 & 1.68 & $<-1.19$ & $<0.16$ & 04 & 18.74 & $(7.2 \mathrm{e}+05)$ & $(1.6 \mathrm{e}+07)$ \\
\hline 2M1207 b & M8 & $52.41 \pm 1.10$ & $<26.24$ & $<27.16$ & 30.07 & $<15$ & 4.00 & 46.00 & $<-4.53$ & $<-3.56$ & 13.71 & 15.78 & $(3.3 e+02)$ & $(3.1 \mathrm{e}+03)$ \\
\hline 30 Ari B b & F6V & $39.43 \pm 1.71$ & 29.53 & $29.36_{-0.03}^{+0.07}$ & 33.83 & 0.16 & 9.88 & 1.00 & 2.09 & 2.31 & 18.35 & 18.93 & $1.4 \mathrm{e}+09$ & $2.3 e+09$ \\
\hline $47 \mathrm{UMa} b$ & G0V & $14.08 \pm 0.13$ & 25.45 & $26.56_{-0.22}^{+0.34}$ & 33.74 & $<15$ & 2.53 & 2.10 & -2.64 & -1.50 & 17.84 & 18.55 & $2.6 \mathrm{e}+04$ & $3.6 \mathrm{e}+05$ \\
\hline 47 UMa c & & & & & & & 0.54 & 3.60 & -3.11 & -1.97 & 17.37 & 18.08 & $8.8 \mathrm{e}+03$ & $1.2 \mathrm{e}+05$ \\
\hline 47 UMa d & & & & & & & 1.64 & 11.60 & -4.12 & -2.98 & 16.36 & 17.06 & $8.4 \mathrm{e}+02$ & $1.2 \mathrm{e}+04$ \\
\hline 51 Peg b & G2IV & $15.36 \pm 0.18$ & $<26.52$ & $<27.86$ & 33.65 & 13.50: & 0.47 & 0.05 & $<1.64$ & $<3.00$ & 21.04: & 21.76: & $(4.9 \mathrm{e}+08)$ & $(1.1 \mathrm{e}+10)$ \\
\hline $55 \mathrm{Cnc} b$ & G8V & $12.53 \pm 0.13$ & 26.65 & $27.49_{-0.42}^{+0.46}$ & 33.39 & 11.19 & 0.82 & 0.12 & 1.08 & 1.98 & 20.28 & 21.05 & $1.4 \mathrm{e}+08$ & $1.1 \mathrm{e}+09$ \\
\hline $55 \mathrm{Cnc} \mathrm{c}$ & & & & & & & 0.17 & 0.24 & 0.44 & 1.34 & 19.64 & 20.41 & $3.1 \mathrm{e}+07$ & $2.4 \mathrm{e}+08$ \\
\hline $55 \mathrm{Cnc} \mathrm{d}$ & & & & & & & 3.84 & 5.77 & -2.32 & -1.42 & 16.88 & 17.65 & $5.4 \mathrm{e}+04$ & $4.2 \mathrm{e}+05$ \\
\hline $55 \mathrm{Cnc}$ e & & & & & & & 0.02 & 0.04 & 2.04 & 2.94 & 21.24 & 22.01 & $1.2 \mathrm{e}+09$ & $9.7 \mathrm{e}+09$ \\
\hline $55 \mathrm{Cnc} \mathrm{f}$ & & & & & & & 0.14 & 0.79 & -0.59 & 0.31 & 18.61 & 19.38 & $2.9 \mathrm{e}+06$ & $2.3 e+07$ \\
\hline$\beta$ Pic b & A6V & $19.28 \pm 0.19$ & 25.63 & $27.01_{-0.39}^{+0.44}$ & 34.49 & $\ldots$ & 8.00 & 12.00 & -3.97 & -2.58 & $\ldots$ & $\ldots$ & $1.2 \mathrm{e}+03$ & $2.9 \mathrm{e}+04$ \\
\hline$\epsilon$ Eridani b & $\mathrm{K} 2 \mathrm{~V}$ & $3.22 \pm 0.01$ & 28.20 & $28.44_{-0.00}^{+0.00}$ & 33.10 & 1.12 & 1.55 & 3.39 & -0.31 & 0.13 & 17.19 & 18.00 & $5.5 \mathrm{e}+06$ & $1.5 \mathrm{e}+07$ \\
\hline GJ 86 b & $\mathrm{K} 1 \mathrm{~V}$ & $10.91 \pm 0.07$ & $<27.42$ & $<29.15$ & 33.16 & 3.59 & 4.01 & 0.11 & $<1.88$ & $<3.63$ & 20.23 & 21.04 & $(8.6 \mathrm{e}+08)$ & $(4.8 \mathrm{e}+10)$ \\
\hline GJ $317 \mathrm{~b}$ & M3.5 & $9.01 \pm 0.97$ & 26.12 & $27.43_{-0.45}^{+0.47}$ & 30.18 & $<15$ & 1.20 & 0.95 & -1.28 & 0.05 & 17.15 & 19.15 & $5.9 \mathrm{e}+05$ & $1.3 \mathrm{e}+07$ \\
\hline GJ 436 b & M2.5 & $10.23 \pm 0.24$ & 25.96 & $26.87_{-0.40}^{-0.45}$ & 31.67 & $<15$ & 0.07 & 0.03 & 1.59 & 2.55 & 20.88 & 22.20 & $4.4 \mathrm{e}+08$ & $4.0 \mathrm{e}+09$ \\
\hline GJ 674 b & M2.5 & $4.54 \pm 0.03$ & 27.72 & $28.01_{-0.17}^{+0.28}$ & 31.54 & 2.27 & 0.04 & 0.04 & 3.09 & 3.56 & 20.42 & 21.86 & $1.4 \mathrm{e}+10$ & $4.1 \mathrm{e}+10$ \\
\hline GJ 876 b & $\mathrm{M} 4 \mathrm{~V}$ & $4.70 \pm 0.05$ & 26.16 & $26.65_{-0.31}^{+0.41}$ & 31.37 & $<15$ & 2.28 & 0.21 & 0.07 & 0.69 & 9.04 & 20.48 & $1.3 e+07$ & $5.5 \mathrm{e}+07$ \\
\hline GJ 876 c & & & & & & & 0.71 & 0.13 & 0.48 & 1.10 & 19.45 & 20.89 & $3.4 \mathrm{e}+07$ & $1.4 \mathrm{e}+08$ \\
\hline GJ $876 \mathrm{~d}$ & & & & & & & 0.02 & 0.02 & 2.07 & 2.69 & 21.04 & 22.48 & $1.3 e+09$ & $5.5 \mathrm{e}+09$ \\
\hline GJ $876 \mathrm{e}$ & & & & & & & 0.05 & 0.33 & -0.34 & 0.28 & 3 & 20.07 & $5.2 \mathrm{e}+06$ & $2.1 \mathrm{e}+07$ \\
\hline GQ Lup b & K7V & $140:$ & 29.45 & $31.05_{-0.45}^{+0.47}$ & 33.36 & 0.17 & 21.50 & 103.00 & -2.02 & -0.41 & 14.16 & 14.87 & $1.1 \mathrm{e}+05$ & $4.3 e+06$ \\
\hline HD $4308 \mathrm{~b}$ & G5V & $21.85 \pm 0.27$ & $<25.94$ & $<27.28$ & 33.57 & $<15$ & 0.04 & 0.12 & $<0.35$ & $<1.71$ & 20.31 & 21.04 & $(2.5 \mathrm{e}+07)$ & $(5.7 \mathrm{e}+08)$ \\
\hline HD 20367 b & G0V & $27.13 \pm 0.79$ & 29.29 & $29.43_{-0.21}^{+0.33}$ & 33.78 & 0.22 & 1.07 & 1.25 & 1.65 & 2.02 & 18.17 & 18.77 & $5.0 \mathrm{e}+08$ & $1.2 \mathrm{e}+09$ \\
\hline HD 27442 b & K2IV & $18.23 \pm 0.17$ & $<26.14$ & $<27.47$ & 34.40 & $<15$ & 1.35 & 1.16 & $<-1.44$ & $<-0.09$ & 18.58: & 19.12: & $(4.1 \mathrm{e}+05)$ & $(9.2 \mathrm{e}+06)$ \\
\hline HD 46375 b & K1IV & $33.41 \pm 1.19$ & 27.16 & $28.49_{-0.45}^{+0.47}$ & 33.48 & 5.22: & 0.25 & 0.04 & 2.49 & 3.84 & 21.19: & 21.93: & $3.5 e+09$ & $7.7 \mathrm{e}+10$ \\
\hline HD 49674 b & G5V & $40.73 \pm 1.89$ & 27.41 & $29.36_{-0.08}^{+0.43}$ & 33.51 & 3.62 & 0.12 & 0.06 & 2.43 & 4.39 & 20.89 & 21.61 & $3.1 \mathrm{e}+09$ & $2.8 \mathrm{e}+11$ \\
\hline HD 50554 b & F8 & $31.03 \pm 0.97$ & $<26.32$ & $<27.66$ & 33.74 & $<15$ & 5.16 & 2.41 & $<-1.89$ & $<-0.53$ & 17.72 & 18.43 & $(1.4 \mathrm{e}+05)$ & $(3.3 e+06)$ \\
\hline HD 52265 b & G0V & $28.07 \pm 0.66$ & 26.89 & $27.72_{-0.28}^{+0.38}$ & 33.86 & 7.80 & 1.05 & 0.50 & 0.05 & 0.93 & 19.11 & 19.78 & $1.3 \mathrm{e}+07$ & $9.7 \mathrm{e}+07$ \\
\hline HD 70642 b & G5IV-V & $28.76 \pm 0.50$ & 26.38 & $27.71_{-0.44}^{+0.47}$ & 33.58 & $<15$ & 2.00 & 3.30 & -2.10 & -0.76 & 17.41: & 18.15: & $8.9 \mathrm{e}+04$ & $2.0 \mathrm{e}+06$ \\
\hline HD 75289 b & G0V & $28.94 \pm 0.47$ & $<25.90$ & $<27.24$ & 33.87 & $<15$ & 0.42 & 0.05 & $<1.13$ & $<2.49$ & 21.19 & 21.87 & $(1.5 \mathrm{e}+08)$ & $(3.4 \mathrm{e}+09)$ \\
\hline HD 93083 b & K3V & $28.90 \pm 0.84$ & 26.90 & $28.36_{-0.46}^{+0.47}$ & 33.19 & 7.77 & 0.37 & 0.48 & 0.09 & 1.56 & 18.98 & 19.79 & $1.4 \mathrm{e}+07$ & $4.1 \mathrm{e}+08$ \\
\hline HD 95089 b & KOIV & $139.08 \pm 0.00$ & $<27.09$ & $<28.43$ & 34.71 & 5.85: & 1.20 & 1.51 & $<-0.72$ & $<0.64$ & 18.46: & 18.90: & $(2.1 \mathrm{e}+06)$ & $(4.9 \mathrm{e}+07)$ \\
\hline HD 99492 b & K2V & $17.99 \pm 1.07$ & 26.55 & $27.85_{-0.45}^{+0.47}$ & 33.11 & 13.01 & 0.11 & 0.12 & 0.92 & 2.24 & 20.14 & 20.98 & $9.3 e+07$ & $2.0 \mathrm{e}+09$ \\
\hline HD 99492 c & & & & & & & 0.36 & 5.40 & -2.36 & -1.04 & 16.85 & 17.70 & $4.9 \mathrm{e}+04$ & $1.0 \mathrm{e}+06$ \\
\hline HD $101930 \mathrm{~b}$ & K1V & $30.50 \pm 0.89$ & 26.05 & $27.00_{-0.28}^{+0.39}$ & 33.27 & $<15$ & 0.30 & 0.30 & -0.36 & 0.64 & 19.41 & 20.21 & $4.9 \mathrm{e}+06$ & $4.9 \mathrm{e}+07$ \\
\hline HD 102195 b & KOV & $28.98 \pm 0.97$ & 28.43 & $29.76_{-0.32}^{+0.41}$ & 33.26 & 0.80 & 0.45 & 0.05 & 3.60 & 4.95 & 20.91 & 21.67 & $4.5 \mathrm{e}+10$ & $1.0 \mathrm{e}+12$ \\
\hline HD 108147 b & $\mathrm{F} 8 / \mathrm{G} 0 \mathrm{~V}$ & $38.57 \pm 1.03$ & 27.39 & $28.67_{-0.45}^{+0.47}$ & 33.85 & 3.74 & 0.26 & 0.10 & 1.92 & 3.23 & 20.48 & 21.14 & $9.4 \mathrm{e}+08$ & $1.9 \mathrm{e}+10$ \\
\hline HD $111232 b$ & G8V & $28.88 \pm 0.67$ & $<26.35$ & $<27.69$ & 33.40 & $<15$ & 6.80 & 1.97 & $<-1.69$ & $<-0.33$ & 17.81 & 18.59 & $(2.3 e+05)$ & $(5.3 e+06)$ \\
\hline HD 114386 b & K3V & $28.04 \pm 1.04$ & 26.53 & $27.40_{-0.34}^{+0.42}$ & 33.02 & 13.41 & 1.24 & 1.65 & -1.36 & -0.43 & 17.85 & 18.72 & $5.0 \mathrm{e}+05$ & $4.1 \mathrm{e}+06$ \\
\hline HD 114762 b & F9V & $40.57 \pm 2.37$ & $<26.51$ & $<27.83$ & 33.76 & 13.73 & 11.68 & 0.36 & $<-0.06$ & $<1.28$ & 19.37 & 20.07 & $(9.9 \mathrm{e}+06)$ & $(2.1 \mathrm{e}+08)$ \\
\hline HD 114783 b & KOV & $20.43 \pm 0.44$ & $<26.54$ & $<27.83$ & 33.19 & 13.17 & 1.00 & 1.20 & $<-1.07$ & $<0.24$ & 18.18 & 19.01 & $(9.6 \mathrm{e}+05)$ & $(2.0 \mathrm{e}+07)$ \\
\hline HD 130322 b & KOV & $29.76 \pm 1.34$ & 27.25 & $28.57_{-0.44}^{+0.47}$ & 33.28 & 4.60 & 1.02 & 0.09 & 1.91 & 3.25 & 20.47 & 21.25 & $9.2 \mathrm{e}+08$ & $2.0 \mathrm{e}+10$ \\
\hline HD 154345 b & G8V & $18.06 \pm 0.18$ & 27.12 & $27.95_{-0.26}^{+0.37}$ & 33.36 & 5.60 & 0.95 & 4.19 & -1.58 & -0.69 & 17.14 & 17.90 & $3.0 \mathrm{e}+05$ & $2.3 e+06$ \\
\hline HD 164922 b & KOV & $21.93 \pm 0.34$ & $<25.74$ & $<27.08$ & 33.44 & $<15$ & 0.36 & 2.11 & $<-2.35$ & $<-1.00$ & 17.77 & 18.53 & $(5.0 \mathrm{e}+04)$ & $(1.1 \mathrm{e}+06)$ \\
\hline HD 179949 b & F8V & $27.05 \pm 0.59$ & 28.38 & $29.52_{-0.29}^{+0.39}$ & 33.84 & 0.85 & 0.95 & 0.05 & 3.63 & 4.80 & 21.15 & 21.78 & $4.8 \mathrm{e}+10$ & $7.0 \mathrm{e}+11$ \\
\hline HD 187123 b & G5 & $47.92 \pm 1.63$ & $<27.24$ & $<28.62$ & 33.78 & 4.67 & 0.52 & 0.04 & $<2.53$ & $<3.93$ & 21.22 & 21.90 & $(3.8 \mathrm{e}+09)$ & $(9.6 \mathrm{e}+10)$ \\
\hline HD 187123 c & & & & & & & 1.99 & 4.89 & $<-1.59$ & $<-0.19$ & 17.11 & 17.78 & $(2.9 \mathrm{e}+05)$ & $(7.3 e+06)$ \\
\hline HD 189733 b & K1-K2 & $19.25 \pm 0.32$ & 28.18 & $28.48_{-0.08}^{+0.18}$ & 33.10 & 1.16 & 1.15 & 0.03 & 3.73 & 4.21 & 21.26 & 22.07 & $6.1 \mathrm{e}+10$ & $1.8 \mathrm{e}+11$ \\
\hline HD $190360 \mathrm{~b}$ & G6IV & $15.89 \pm 0.16$ & $<26.35$ & $<27.69$ & 33.65 & $<15$ & 1.50 & 3.92 & $<-2.29$ & $<-0.93$ & 17.28: & 18.00: & $(5.8 \mathrm{e}+04)$ & $(1.3 e+06)$ \\
\hline HD 190360 c & & & & & & & 0.06 & 0.13 & $<0.69$ & $<2.05$ & 20.25: & 20.98: & $(5.5 \mathrm{e}+07)$ & $(1.3 e+09)$ \\
\hline HD 195019 b & G3IV-V & $37.36 \pm 1.24$ & $<26.23$ & $<27.57$ & 33.89 & $<15$ & 3.70 & 0.14 & $<0.50$ & $<1.86$ & 20.24: & 20.92: & $(3.5 \mathrm{e}+07)$ & $(8.1 \mathrm{e}+08)$ \\
\hline HD 209458 b & G0V & $47.08 \pm 2.22$ & $<26.40$ & $<27.74$ & 33.78 & $<15$ & 0.71 & 0.05 & $<1.60$ & $<2.96$ & 21.14 & 21.84 & $(4.5 \mathrm{e}+08)$ & $(1.0 \mathrm{e}+10)$ \\
\hline HD $216435 b$ & G0V & $33.29 \pm 0.81$ & 27.74 & $28.99_{-0.44}^{+0.47}$ & 34.14 & 2.22 & 1.26 & 2.56 & -0.53 & 0.75 & 17.78 & 18.35 & $3.3 \mathrm{e}+06$ & $6.3 e+07$ \\
\hline
\end{tabular}


Table 6. continued.

\begin{tabular}{|c|c|c|c|c|c|c|c|c|c|c|c|c|c|c|}
\hline Planet name & $\begin{array}{l}\text { Sp. type } \\
\text { (star) }\end{array}$ & $\begin{array}{l}\text { Stellar distance } \\
\text { (pc) }\end{array}$ & $\begin{array}{c}\log L_{\mathrm{X}} \\
\left(\mathrm{erg} \mathrm{s}^{-1}\right)\end{array}$ & $\begin{array}{l}\log L_{\mathrm{EUV}} \\
\left(\mathrm{erg} \mathrm{s}^{-1}\right)\end{array}$ & $\begin{array}{l}\log L_{\mathrm{bol}} \\
\left(\mathrm{erg} \mathrm{s}^{-1}\right)\end{array}$ & $\begin{array}{l}\text { age } \\
(\mathrm{Gyr})\end{array}$ & $\begin{array}{c}M_{\mathrm{p}} \sin i \\
\left(m_{\mathrm{J}}\right)\end{array}$ & $\begin{array}{c}a_{\mathrm{p}} \\
(\mathrm{au})\end{array}$ & $\begin{array}{l}\log F_{\mathrm{X}} \\
\quad\left(\mathrm{erg} \mathrm{s}^{-1}\right.\end{array}$ & $\begin{array}{l}\log F_{\mathrm{XUV}} \\
\left.{ }^{-1} \mathrm{~cm}^{-2}\right)\end{array}$ & \multicolumn{2}{|c|}{$\left(\mathrm{erg} \mathrm{cm}^{-2}\right)$} & \multicolumn{2}{|c|}{$\left(\mathrm{g}^{2} \mathrm{~s}^{-1} \mathrm{~cm}^{-3}\right)^{c}$} \\
\hline HD $216437 b$ & G4IV-V & $26.52 \pm 0.41$ & 26.62 & $27.47_{-0.35}^{+0.42}$ & 33.92 & 11.68: & 1.82 & 2.32 & -1.56 & -0.65 & 17.80: & 18.47: & $3.1 \mathrm{e}+05$ & $2.5 \mathrm{e}+06$ \\
\hline HD $217107 \mathrm{~b}$ & G8IV & $19.72 \pm 0.29$ & $<25.50$ & $<26.84$ & 33.64 & $<15$ & 1.33 & 0.07 & $<0.32$ & $<1.68$ & 20.74: & 21.46: & $(2.4 \mathrm{e}+07)$ & $(5.4 \mathrm{e}+08)$ \\
\hline HD $217107 \mathrm{c}$ & & & & & & & 2.49 & 5.27 & $<-3.40$ & $<-2.03$ & 17.02: & 17.75: & $(4.5 e+03)$ & $(1.0 \mathrm{e}+05)$ \\
\hline HD 218566 b & $\mathrm{K} 3 \mathrm{~V}$ & $29.94 \pm 1.07$ & 27.04 & $28.35_{-0.44}^{+0.47}$ & 33.13 & 6.28 & 0.21 & 0.69 & -0.08 & 1.25 & 18.64 & 19.47 & $9.3 e+06$ & $2.0 \mathrm{e}+08$ \\
\hline HD 330075 b & G5 & $50.20 \pm 3.75$ & 26.51 & $27.36_{-0.42}^{+0.46}$ & 33.26 & 13.80 & 0.62 & 0.04 & 1.88 & 2.79 & 21.18 & 21.99 & $8.5 \mathrm{e}+08$ & $6.9 e+09$ \\
\hline HR 8799 b & $\mathrm{A} 5 \mathrm{~V}$ & $39.94 \pm 1.36$ & 28.02 & $29.37_{-0.45}^{+0.47}$ & 34.26 & $\ldots$ & 7.00 & 68.00 & -3.09 & -1.72 & $\ldots$ & $\ldots$ & $9.0 \mathrm{e}+03$ & $2.1 \mathrm{e}+05$ \\
\hline HR 8799 c & & & & & & & 10.00 & 38.00 & -2.59 & -1.22 & $\ldots$ & $\ldots$ & $2.9 \mathrm{e}+04$ & $6.8 \mathrm{e}+05$ \\
\hline HR $8799 \mathrm{~d}$ & & & & & & & 10.00 & 24.00 & -2.19 & -0.82 & $\ldots$ & $\ldots$ & $7.3 e+04$ & $1.7 \mathrm{e}+06$ \\
\hline HR 8799 e & & & & & & & 9.00 & 14.50 & -1.75 & -0.38 & $\ldots$ & $\ldots$ & $2.0 \mathrm{e}+05$ & $4.7 e+06$ \\
\hline$\mu$ Ara b & G3IV-V & $15.28 \pm 0.19$ & $<25.99$ & $<27.32$ & 33.83 & $<15$ & 1.68 & 1.50 & $<-1.81$ & $<-0.46$ & 18.15: & 18.84: & $(1.7 \mathrm{e}+05)$ & $(3.9 \mathrm{e}+06)$ \\
\hline$\mu$ Ara c & & & & & & & 0.03 & 0.09 & $<0.62$ & $<1.97$ & 20.59: & 21.28: & $(4.7 e+07)$ & $(1.1 \mathrm{e}+09)$ \\
\hline$\mu$ Ara d & & & & & & & 0.52 & 0.92 & $<-1.39$ & $<-0.04$ & 18.58: & 19.27: & $(4.6 \mathrm{e}+05)$ & $(1.0 \mathrm{e}+07)$ \\
\hline$\mu$ Ara e & & & & & & & 1.81 & 5.24 & $<-2.90$ & $<-1.55$ & 17.07: & 17.76: & $(1.4 \mathrm{e}+04)$ & $(3.2 \mathrm{e}+05)$ \\
\hline NGC 24233 b & & $766:$ & $<29.27$ & $<34.91$ & 35.54 & 0.23 & 10.60 & 2.10 & $<1.18$ & $<6.82$ & 18.45 & 18.64 & $(1.7 \mathrm{e}+08)$ & $(7.4 \mathrm{e}+13)$ \\
\hline Pollux b & KOIII & $10.34 \pm 0.09$ & 27.13 & $28.38_{-0.10}^{+0.21}$ & 35.19 & $\ldots$ & 2.90 & 1.69 & -0.77 & 0.50 & $\ldots$ & $\ldots$ & $1.9 \mathrm{e}+06$ & $3.6 e+07$ \\
\hline$\tau$ Boo b & F7V & $15.60 \pm 0.17$ & 28.95 & $29.40_{-0.28}^{+0.39}$ & 34.06 & 0.37 & 3.90 & 0.05 & 4.18 & 4.76 & 21.19 & 21.72 & $1.7 \mathrm{e}+11$ & $6.4 \mathrm{e}+11$ \\
\hline$v$ And $\mathrm{b}$ & F8V & $13.47 \pm 0.13$ & 27.56 & $28.73_{-0.41}^{+0.46}$ & 34.11 & 2.88 & 0.69 & 0.06 & 2.57 & 3.77 & 21.04 & 21.63 & $4.2 \mathrm{e}+09$ & $6.6 e+10$ \\
\hline$v$ And c & & & & & & & 14.57 & 0.86 & 0.25 & 1.44 & 18.72 & 19.30 & $2.0 \mathrm{e}+07$ & $3.1 \mathrm{e}+08$ \\
\hline$v$ And $\mathrm{d}$ & & & & & & & 10.19 & 2.55 & -0.70 & 0.50 & 17.77 & 18.36 & $2.3 e+06$ & $3.6 e+07$ \\
\hline$v$ And $\mathrm{e}$ & & & & & & & 1.06 & 5.25 & -1.32 & -0.13 & 17.15 & 17.73 & $5.3 e+05$ & $8.4 \mathrm{e}+06$ \\
\hline
\end{tabular}

Notes. ${ }^{(a)}$ Planet data from The Extrasolar Planets Encyclopedia (http://exoplanet. eu). ${ }^{(c)} 1 \mathrm{MJ}_{\mathrm{J}} \mathrm{Gyr}^{-1}=6.02 \times 10^{13} \mathrm{~g} \mathrm{~s}^{-1}$.

expected that thermal losses are dominant. The mass loss rate through thermal losses in a planet with an atmosphere mostly composed of hydrogen is defined by Eq. (2). To account for the energy budget in the XUV band, we need to know the radiation that is absorbed by neutral hydrogen, i.e., photons with $\lambda<912 \AA$. In Paper I we considered only the X-rays photons as a proxy of the whole XUV radiation. It is therefore necessary to test whether the conclusions achieved hold for the whole XUV range as well. With the whole energy budget we can make a first estimate of the total mass lost through thermal losses for the planets in the sample with known density (see below). Figures 4 and 5 show the distribution of planet masses $\left(M_{\mathrm{P}} \sin i\right)$ with the X-ray flux at the planet orbit (directly measured) and the XUV flux that we calculated after modeling the corona and transition region. A dashed line $\left(\log F_{\mathrm{X}}=3-0.5 M_{\mathrm{p}} \sin i\right)$ is plotted in Fig. 4 to indicate the "erosion line", proposed in Paper I to separate what could be a regime of fast erosion from a slower erosion phase, based on the observed distribution. Figure 5 is a direct indication of the current mass loss rate in exoplanets, assuming that thermal losses are dominant and the density is the same for all planets (according to Eq. (2)). Regrettably, we know the planet density of only four planets in our sample (Table 7). Rocky planets $\left(\rho \sim 5 \mathrm{~g} \mathrm{~cm}^{-3}\right)$ are not supposed to be affected in the same manner (a gaseous planet like Jupiter has $\rho=1.24$ ). We marked in the plot the XUV flux that would have arrived at the Earth's orbit at an age of $\sim 100 \mathrm{Myr}$ and $\sim 1 \mathrm{Gyr}$. To calculate this flux, we used two young solar analogs, $\kappa$ Cet and EK Dra, as explained in Paper I. The emission in the EUV range was calculated by Sanz-Forcada et al. (in prep.) extrapolating from a coronal model determined with high-resolution spectra.

A better way to measure the long-term effects of the radiation is to calculate the accumulated XUV flux at the orbit of the planet. To do that, we need to know how coronal radiation evolves with time. Several laws have been reported in the past, mostly devoted to explain the coronal history of the

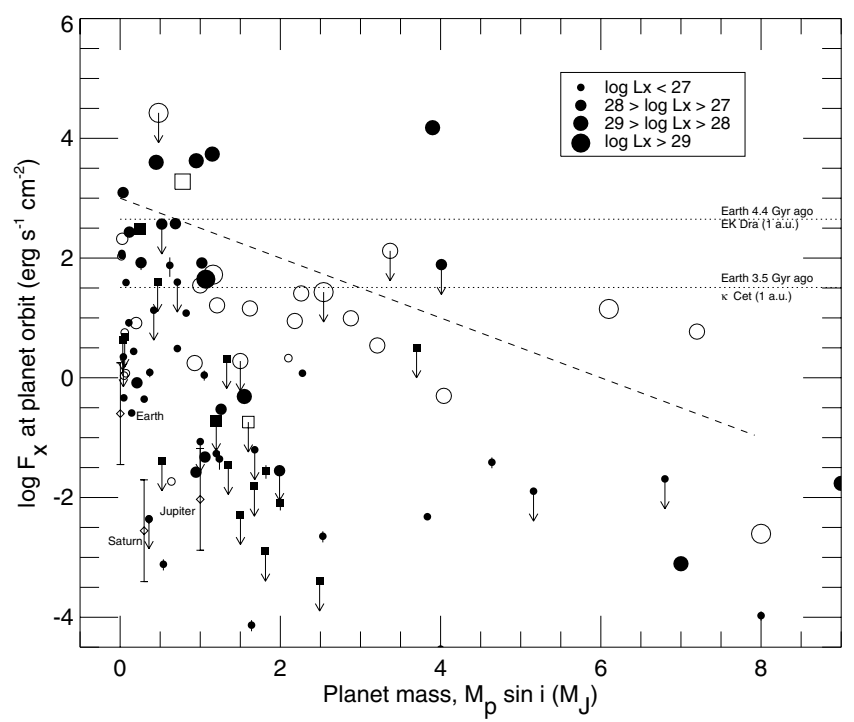

Fig. 4. Distribution of planetary masses $\left(M_{\mathrm{p}} \sin i\right)$ with X-ray flux at the planet orbit. Filled symbols (squares for subgiants, circles for dwarfs) are XMM-Newton and Chandra data. Arrows indicate upper limits. Open symbols are ROSAT data without error bars. Diamonds represent Jupiter, Saturn, and the Earth. The dashed line marks the "erosion line" that might separate the phases of strong and weak evaporation (Paper I). Dotted lines indicate the X-ray flux of the younger Sun at $1 \mathrm{au}$.

Sun as a law of the kind $L_{\mathrm{X}} \sim t^{\alpha}$, calibrated with $\mathrm{G}$ dwarfs. Some examples are Maggio et al. (1987, $\alpha=-1.5)$, Ayres (1997, $\alpha=-1.74)$, Guedel et al. (1997, $\alpha=-1.5)$, Ribas et al. $(2005, \alpha=-1.27$ or -1.92 , for $1-20 \AA$ and $20-100 \AA$ respectively), Penz et al. (2008, $\alpha=-1.69)$, and Penz \& Micela (2008, $\alpha=-1.34$, calibrated with $\mathrm{M}$ dwarfs). We use the law 


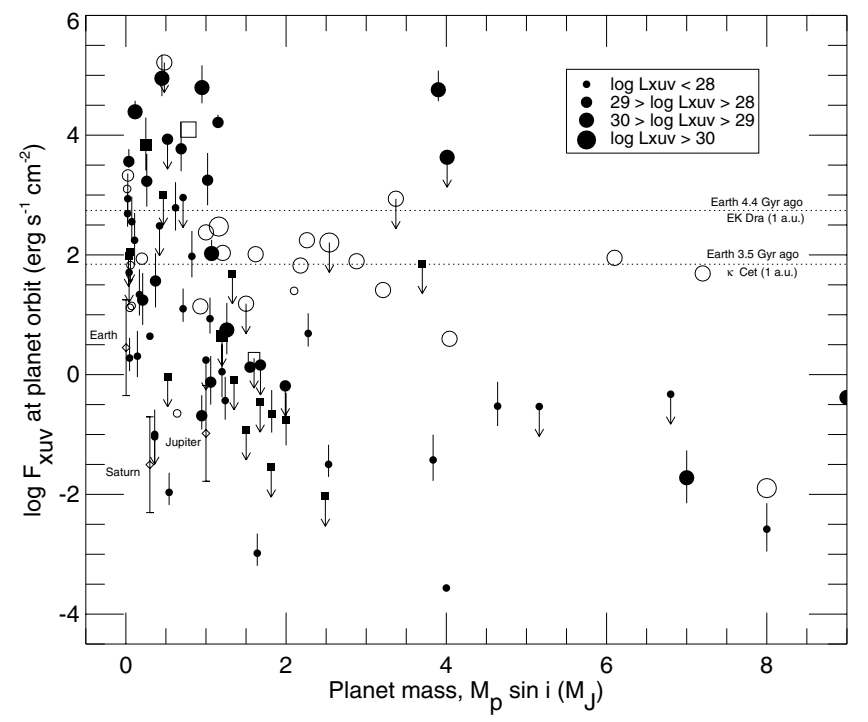

Fig. 5. Distribution of planetary masses $\left(M_{\mathrm{p}} \sin i\right)$ with XUV flux at the planet orbit. Symbols as in Fig. 4 Error bars indicate the limits in EUV flux calculated with the models. Dotted lines indicate the X-ray flux of the younger Sun at 1 au. Note that mass loss rates increase with the XUV flux.

by Garcés et al. (in prep.; see also Garcés et al. 2011), calibrated with late $\mathrm{F}$ to early $\mathrm{M}$ dwarfs:

$$
\begin{aligned}
& L_{\mathrm{X}}=6.3 \times 10^{-4} L_{\mathrm{bol}} \quad\left(\tau<\tau_{i}\right) \\
& L_{\mathrm{X}}=1.89 \times 10^{28} \tau^{-1.55} \quad\left(\tau>\tau_{i}\right) \text {, }
\end{aligned}
$$

with $\tau_{i}=2.03 \times 10^{20} L_{\text {bol }}^{-0.65} . L_{\mathrm{X}}$ and $L_{\mathrm{bol}}$ are in erg s${ }^{-1}$, and $\tau$ is the age in Gyr. The $\tau_{i}$ parameter marks the typical change from saturation regime to an inverse proportionality between $L_{\mathrm{X}} / L_{\mathrm{bol}}$ and rotation period (e.g. Pizzolato et al. 2003). The relation shows a similar behavior to former calibrations, but it can be applied to a wider range of stellar masses. We use Eq. (5) to calculate the stellar age (Table 6), with the caveat that there is an uncertainty of about an order of magnitude in the $L_{X}$ levels of stars of the same spectral type and age (Penz et al. 2008; Penz \& Micela 2008), and that the solar cycle in X-rays spans as much as $1.7 \mathrm{dex}$ in $L_{X}$ (Orlando et al. 2001). We consider a formal upper limit of the age at $15 \mathrm{Gyr}$ (current estimate of the age of the Universe is $13.7 \mathrm{Gyr}$, Bennett et al. 2003). This method is not optimal for the age determination of a star, but it is more appropriate if we aim to know the "X-ray age" in the evolution of the coronal emission of a given star.

The accumulated X-ray and XUV flux at the planet orbit (Table 6, Fig. 6) is calculated using Eqs. (4) and (5), between $20 \mathrm{Myr}$ and the present. Most protoplanetary disks would have dissipated after $20 \mathrm{Myr}$. We do not calculate the age for giants and A-type stars, and mark the subgiants with different symbols because it is not known whether they follow the same relation, but sometimes the determination of the star as dwarf or subgiant is not precise. Only planets with $M \sin i<9 M_{\mathrm{J}}$ are considered for further calculations. According to Eq. (2), Fig. 6 is a direct indication of the total mass lost to date, assuming same density for all planets. A planet like $\tau$ Boo b, with $10^{21.7} \mathrm{erg} \mathrm{cm}^{-2} \mathrm{ac}-$ cumulated in the XUV band during $350 \mathrm{Myr}$, would have lost $\sim 0.04 M_{\mathrm{J}}$ if the density is $1 \mathrm{~g} \mathrm{~cm}^{-3}$, but as much as $\sim 0.7 M_{\mathrm{J}}$ if the density is $0.1 \mathrm{~g} \mathrm{~cm}^{-3}$, among the lowest observed in exoplanets.
Table 7. Mass lost in planets with known density.

\begin{tabular}{lcccccccc}
\hline \hline Planet name & $\begin{array}{c}R_{\mathrm{P}} \\
\left(R_{\mathrm{J}}\right)\end{array}$ & $\begin{array}{c}M_{\mathrm{P}} \\
\left(M_{\mathrm{J}}\right)\end{array}$ & $\begin{array}{c}\rho \\
\left(\mathrm{g} \mathrm{cm}^{-3}\right)\end{array}$ & $\begin{array}{c}M_{*} \\
\left(M_{\odot}\right)\end{array}$ & $\begin{array}{c}a_{\mathrm{P}} \\
(\mathrm{au})\end{array}$ & $K^{a}$ & $\begin{array}{c}M_{\text {lost }}{ }^{b} \\
(1)\end{array}$ & $\begin{array}{c}\left(M_{\mathrm{J}}\right) \\
(2)\end{array}$ \\
\hline GJ 436 b & 0.365 & 0.0737 & 1.88 & 0.452 & 0.0289 & 0.76 & 0.06 & 0.08 \\
HD 189733 b & 1.151 & 1.15 & 0.94 & 0.8 & 0.0314 & 0.67 & 0.11 & 0.11 \\
HD 209458 b & 1.38 & 0.714 & 0.34 & 1.0 & 0.0475 & 0.65 & 0.18 & 0.17 \\
2M1207 A b & 1.5 & 4.0 & 1.47 & 0.025 & 46 & 1.00 & $2 \mathrm{e}-8$ & $2 \mathrm{e}-8$ \\
\hline
\end{tabular}

Notes. ${ }^{(a)} K$ parameter to account for the Roche lobe effects as in Eq. (7).

(b) Mass lost since $20 \mathrm{Myr}$ old, including Roche lobe effects and thermal losses. (1) is for constant density; (2) with $M-R$ relation as explained in the text.

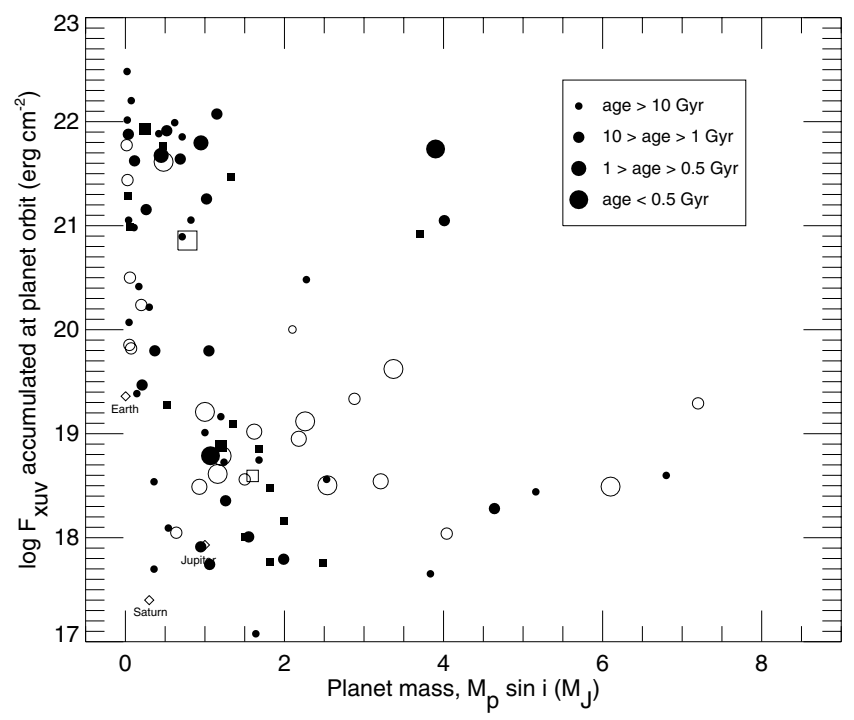

Fig. 6. Distribution of planetary masses $\left(M_{\mathrm{p}} \sin i\right)$ with the XUV flux accumulated at the planet orbit since an age of $20 \mathrm{Myr}$ to the present day (see text). Symbols as in Fig. 4. Note that increasing XUV flux accumulated indicates a larger mass lost to date.

\subsection{Accumulated effects in planets with known density}

It is expected that the accumulated effects of the XUV radiation result in a population of close-in planets with less massive planets, unless they have higher densities, or they simply have little or no atmosphere. The current distribution of planets with known density is dominated by close-in planets, and therefore it has an appropriate bias to test these effects, even if we do not know the radiation of their parent stars. It is remarkable to observe in this distribution that there are no massive planets with low density at short distances of the star (Paper I). We do not know now if this is also applicable for planets at farther distances; that would indicate that this is an effect of planet formation. Theoretical models (e.g. Guillot 2005; Fortney et al. 2007) indicate that irradiated planets might have a nearly constant radius for planets with $M_{\mathrm{P}} \gtrsim 1 M_{\mathrm{J}}$, explaining the increasing density with mass in Fig. 3 of Paper I. The radius-mass relation of the same sample supports this idea (Fig. 7), although actual values of planet radius are slightly higher on average than Fig. 10 of Guillot (2005). This empirical relation can be defined as follows:

$$
\begin{array}{ll}
R_{\mathrm{P}}=(0.15 \pm 0.07)+(4.1 \pm 3.0) M_{\mathrm{P}} & \left(M_{\mathrm{P}}<0.05\right) \\
R_{\mathrm{P}}=(0.29 \pm 0.11)+(2.45 \pm 0.36) M_{\mathrm{P}} & \left(0.05<M_{\mathrm{P}}<0.5\right) \\
R_{\mathrm{P}}=(1.23 \pm 0.05)+(0.00 \pm 0.02) M_{\mathrm{P}} & \left(0.5<M_{\mathrm{P}}<4.5\right) .(6)
\end{array}
$$

With the estimate of the total XUV flux it is possible now to check the mass loss history of the few planets in our sample with known density (we exclude the A5V star HR 8799). While 
2M1207 b suffers basically no erosion (Table 6), the other three planets (GJ 436 b, HD 189733 b, HD 209458 b) show that strong mass loss takes place. As we know the stellar and planet parameters for these cases, we can also calculate the mass loss through the Roche lobe point. The formula in Eq. (1) would be then

$\dot{M}_{\mathrm{XUV}} \sim \frac{3 F_{\mathrm{XUV}}}{4 \mathrm{G} K \rho}$

where the $K$ parameter is defined as a function of $R_{\mathrm{P}}, M_{\mathrm{P}}$ (planet radius and mass), $a_{\mathrm{P}}$ (semimajor axis) and $M_{*}$ (stellar mass), as described in Erkaev et al. (2007). Table 7 lists the parameters used in the calculation. Note that Erkaev et al. (2007) calculates the wrong values of $K$, likely because of a mistake in the substitution of the units used to determine the Roche lobe distance, overestimating the mass losses through the Roche lobe. Figure 8 represents the mass loss history, assuming that planet density remains constant and only thermal losses are eroding the atmosphere. We consider also the hypothesis of mass losses following the same trend (constant radius for HD 209458 b, HD 189733 b and 2M1207 A b, $R_{\mathrm{P}} \sim 2.45 M_{\mathrm{P}}$ for GJ 436) found in the massradius relation of close-in planets (Eq. (6)), which better agrees with theoretical models. Future development of Eq. (7) should give a more accurate view of the thermal losses. In this sense, Lammer et al. (in prep.; see also Lammer et al. 2009) multiply Eq. (7) by the heating efficiency (about 10-25\%). This would imply much lower effects of evaporation, but it is insufficient to justify the mass loss rate measured in HD 209458 b (see below). Other models include different effects of EUV radiation in the atmosphere and infrared cooling (Yelle 2004; Tian et al. 2005; García Muñoz 2007; Murray-Clay et al. 2009), which usually yields lower escape rates than Eq. (7). Those models are not easy to test with our sample, and they do not consider the effects of X-rays.

\section{Discussion}

The distribution of planetary masses with X-ray flux received seems to indicate that planets have lost mass in their first stages. Figure 4 can be interpreted in the same manner as an HR diagram: the lack of planets in a given area of the diagram indicates that they spend only a short time in that phase, while the accumulation of planets in other areas indicates that they spend a long time in that position. We can divide the diagram into four boxes based on the mass (at $1.5 M_{\mathrm{J}}$ ) and the X-ray flux at the planet orbit (at $\left.\log F_{X}=2.15\right)$. Only 1 out of $12(8 \%)$ of the planets with high flux have a high mass, while 38 out of $84(45 \%)$ of those receiving lower fluxes have a high mass (up to $9 M_{\mathrm{J}}$ in this diagram). Therefore there is a notable absence of high mass planets suffering high flux levels. The same exercise applies to Fig. 5.

To explain this distribution, we propose three alternatives: (i) an observational bias; (ii) an effect of planet formation; and (iii) a physical phenomenon that moves the planets from their original positions in the diagram. The observational biases (discussed in Paper I) easily explain the lack of planets in the lower left corner of the diagram, corresponding to low mass planets at long distances from the star. But the same biases should lead to more planets in the upper right corner, where no planets are found. The second possibility, the effects of planet formation, cannot be easily identified at the present level of knowledge. Simulations carried out by, e.g., Mordasini et al. (2009) do not cover planets with $a_{\mathrm{P}}<0.1 \mathrm{au}$, and more recent simulations (Benítez-Llambay et al. 2011) might justify only the gap observed in the mass-distance diagram at $M \lesssim 1 M_{\mathrm{J}}$ and

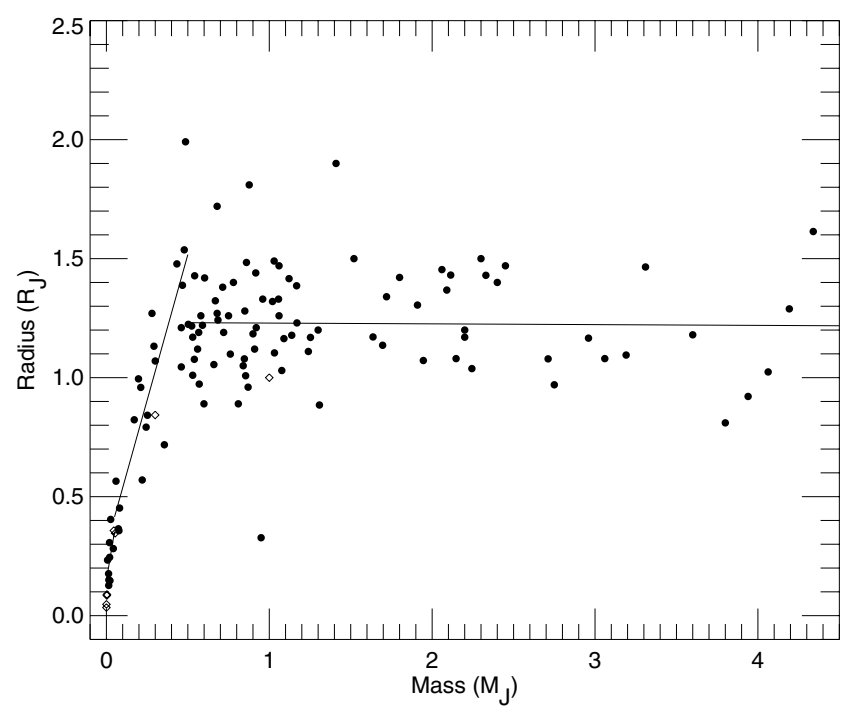

Fig. 7. Mass-radius relation in the sample of exoplanets (8 Mar. 2011) of known radius (filled circles). Open diamonds represent the solar system planets. Solid lines indicate the least-squares fit to the data (see text).

$a_{\mathrm{P}} \lesssim 0.04 \mathrm{au}$. The effects of X-rays in an eventual photoevaporation of the protoplanetary disk has been analyzed by Drake et al. (2009) and Owen et al. (2011). If planet formation favors low mass planets close to the star, Figs. 4 and 5 just reflect a lack of high mass population at short distances $\left(F_{\mathrm{X}} \propto d^{-2}\right)$. However, the distribution of density with mass at short distance of the star (Paper I) reveals that this type of population does exist (32\% of the 120 planets currently in the sample have $M>1.5 M_{\mathrm{J}}$ ), but they have an increasing density with mass, an important detail also for (iii). Finally, it is possible that one or several physical processes related to the XUV emission (and X-rays as a proxy) are eroding the atmospheres of planets at close distances to the star, yielding in the long term an uneven distribution of masses with $F_{\mathrm{X}}$. Equation (2) also indicates that the planet density provides protection against thermal losses, consistent with the observed trend in the mass-density diagram just mentioned. The distribution of masses with the XUV flux accumulated over time (Fig. 6) additionally supports the interpretation of mass losses as the effect of XUV irradiation, either by thermal or non-thermal effects: most massive exoplanets that were initially exposed to high radiation would now have less than $1.5 M_{\mathrm{J}}$.

The upper limit of the hydrogen mass loss rate we calculated for HD $209458 \mathrm{~b}\left(1 \times 10^{10} \mathrm{~g} \mathrm{~s}^{-1}\right)$ is consistent with the values of $\sim 10^{10} \mathrm{~g} \mathrm{~s}^{-1}$ (Vidal-Madjar et al. 2003) interpreted by the authors as hydrogen escaping the planet atmosphere, and with the calculation of $\sim 8 \times 10^{10} \mathrm{~g} \mathrm{~s}^{-1}$ extrapolated from $\mathrm{C}_{\text {II }}$ line absorption by Linsky et al. (2010). Similarly our calculation for HD $189733\left(2 \times 10^{11} \mathrm{~g} \mathrm{~s}^{-1}\right)$ is consistent with the value of $\sim 10^{11} \mathrm{~g} \mathrm{~s}^{-1}$ estimated by Lecavelier Des Etangs et al. (2010). Additional support for our interpretation is found if we look at the chromosphere: Hartman (2010) finds that there is a correlation between chromospheric activity and planet surface gravity. Contrary to the statement by Scharf (2010), this relation supports our conclusions, because denser planets also have a stronger surface gravity. Therefore, the accumulated effects of erosion over time favor a resulting distribution with denser (and with higher surface gravity) planets close to active stars.

The presence of an "erosion line" (Fig. 4) that might separate the stages of strong and weak evaporation (Paper I) cannot be precisely quantified until we have a large sample of planets with 


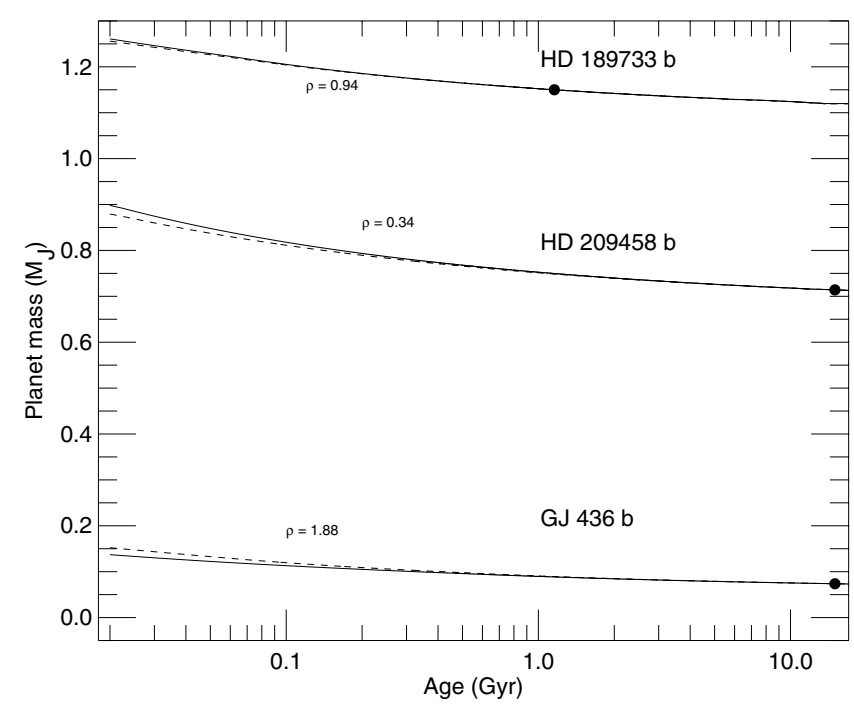

Fig. 8. Time evolution of planetary mass, assuming thermal and Roche lobe losses. Planet density is indicated. Solid lines indicate evolution under constant density conditions, while dashed lines consider the radius-mass relation as explained in the text (constant radius if $\left.M_{\mathrm{P}}>0.5 M_{\mathrm{J}}\right)$. Current age is marked with solid circles.

known density and X-ray measurements of their parent stars. It is possible that such a line separates the phase of heavy erosion of a planet from that of slow or no erosion. The establishment of such a line would be interesting for future works to test whether a planet is still suffering strong erosion. Planets above the line are potential targets for detecting atmospheric features.

\section{Conclusions}

The bulk of evidence supports the effects of erosion on planet atmospheres as an effect of XUV radiation. The accumulated effects of evaporation by radiation yield a population of only low mass planets exposed to a currently high XUV radiation. This interpretation is also supported by the lack of low density massive planets among the close-in planets population. We used a simple physical model to test the observed distribution. This model assumes that mass loses are controlled by the thermal evaporation through the XUV radiation absorbed in the atmosphere. In the four planets of the sample with known density we reconstructed the mass loss history, starting at a stellar age of $20 \mathrm{Myr}$, including thermal losses and losses through the Roche lobe. The density protects the planet atmosphere from further losses, but in a low density planet such as HD $209458 \mathrm{~b}$, up to $\sim 0.2 M_{\mathrm{J}}$ have been lost according to this model. Future model developments should take into account nonthermal losses, the role of planetary magnetic field, and the impact of different atmospheric composition. This research will benefit from the future inclusion of a larger population of planets with known density and X-ray stellar emission.

Acknowledgements. J.S.F. and D.G.A. acknowledge support from the Spanish MICINN through grant AYA2008-02038 and the Ramón y Cajal Program ref. RYC-2005-000549. I.R. acknowledges support from the Spanish MICINN via grant AYA2006-15623-C02-01. G.M. acknowledges financial contribution from PRIN/INAF (P.I.: Lanza). This research has made use of the NASA's High Energy Astrophysics Science Archive Research Center (HEASARC) and the public archives of XMM-Newton and Chandra. We are grateful to the anonymous referee and to the editor, T. Guillot, for the careful reading of and useful comments on the manuscript.

\section{References}

Anders, E., \& Grevesse, N. 1989, Geochim. Cosmochim. Acta, 53, 197

Asplund, M., Grevesse, N., \& Sauval, A. J. 2005, in Cosmic Abundances as Records of Stellar Evolution and Nucleosynthesis, ed. T. G. Barnes, III, \& F. N. Bash, ASP Conf. Ser., 336, 25

Ayres, T. R. 1997, J. Geophys. Res., 102, 1641

Baraffe, I., Selsis, F., Chabrier, G., et al. 2004, A\&A, 419, L13

Benítez-Llambay, P., Masset, F., \& Beaugé, C. 2011, A\&A, 528, A2

Bennett, C. L., Halpern, M., Hinshaw, G., et al. 2003, ApJS, 148, 1

Cecchi-Pestellini, C., Ciaravella, A., Micela, G., \& Penz, T. 2009, A\&A, 496, 863

Cox, A. N. 2000, Allen's astrophysical quantities, ed. A. N. Cox

Drake, J. J., Ercolano, B., Flaccomio, E., \& Micela, G. 2009, ApJ, 699, L35

Dupree, A. K., Brickhouse, N. S., Doschek, G. A., Green, J. C., \& Raymond, J. C. 1993, ApJ, 418, L41

Erkaev, N. V., Kulikov, Y. N., Lammer, H., et al. 2007, A\&A, 472, 329

Flower, P. J. 1996, ApJ, 469, 355

Fortney, J. J., Marley, M. S., \& Barnes, J. W. 2007, ApJ, 659, 1661

Garcés, A., Catalán, S., \& Ribas, I. 2011, A\&A, 531, A7

García Muñoz, A. 2007, Planet. Space Sci., 55, 1426

Guedel, M., Guinan, E. F., \& Skinner, S. L. 1997, ApJ, 483, 947

Guillot, T. 2005, Annu. Rev. Earth Planet. Sci., 33, 493

Hartman, J. D. 2010, ApJ, 717, L138

Houck, J. C., \& Denicola, L. A. 2000, in Astronomical Data Analysis Software and Systems IX, ed. N. Manset, C. Veillet, \& D. Crabtree, ASP Conf. Ser., 216,591

Huenemoerder, D. P., Canizares, C. R., Drake, J. J., \& Sanz-Forcada, J. 2003, ApJ, 595, 1131

Huensch, M., Schmitt, J. H. M. M., \& Voges, W. 1998, A\&AS, 132, 155

Jaritz, G. F., Endler, S., Langmayr, D., et al. 2005, A\&A, 439, 771

Kashyap, V. L., Drake, J. J., \& Saar, S. H. 2008, ApJ, 687, 1339

Lammer, H., Selsis, F., Ribas, I., et al. 2003, ApJ, 598, L121

Lammer, H., Odert, P., Leitzinger, M., et al. 2009, A\&A, 506, 399

Lecavelier Des Etangs, A. 2007, A\&A, 461, 1185

Lecavelier des Etangs, A., Vidal-Madjar, A., McConnell, J. C., \& Hébrard, G. 2004, A\&A, 418, L1

Lecavelier Des Etangs, A., Ehrenreich, D., Vidal-Madjar, A., et al. 2010, A\&A, 514, A72

Linsky, J. L., Yang, H., France, K., et al. 2010, ApJ, 717, 1291

Maggio, A., Sciortino, S., Vaiana, G. S., et al. 1987, ApJ, 315, 687

Maggio, A., Sanz-Forcada, J., \& Scelsi, L. 2011, A\&A, 527, A144

Mordasini, C., Alibert, Y., \& Benz, W. 2009, A\&A, 501, 1139

Murray-Clay, R. A., Chiang, E. I., \& Murray, N. 2009, ApJ, 693, 23

Orlando, S., Peres, G., \& Reale, F. 2001, ApJ, 560, 499

Owen, J. E., Ercolano, B., \& Clarke, C. J. 2011, MNRAS, 412, 13

Penz, T., \& Micela, G. 2008, A\&A, 479, 579

Penz, T., Micela, G., \& Lammer, H. 2008, A\&A, 477, 309

Pizzolato, N., Maggio, A., Micela, G., Sciortino, S., \& Ventura, P. 2003, A\&A, 397, 147

Poppenhaeger, K., Robrade, J., \& Schmitt, J. H. M. M. 2010, A\&A, 515, A98

Raassen, A. J. J., Ness, J., Mewe, R., et al. 2003, A\&A, 400, 671

Ribas, I., Guinan, E. F., Güdel, M., \& Audard, M. 2005, ApJ, 622, 680

Sanz-Forcada, J., \& Micela, G. 2002, A\&A, 394, 653

Sanz-Forcada, J., Brickhouse, N. S., \& Dupree, A. K. 2002, ApJ, 570, 799

Sanz-Forcada, J., Brickhouse, N. S., \& Dupree, A. K. 2003a, ApJS, 145, 147

Sanz-Forcada, J., Maggio, A., \& Micela, G. 2003b, A\&A, 408, 1087

Sanz-Forcada, J., Favata, F., \& Micela, G. 2004, A\&A, 416, 281

Sanz-Forcada, J., García-Álvarez, D., Velasco, A., et al. 2010a, in IAU Symp. 264, ed. A. G. Kosovichev, A. H. Andrei, \& J.-P. Roelot, 478

Sanz-Forcada, J., Ribas, I., Micela, G., et al. 2010b, A\&A, 511, L8

Scharf, C. A. 2010, ApJ, 722, 1547

Schmitt, J. H. M. M., Fleming, T. A., \& Giampapa, M. S. 1995, ApJ, 450, 392

Smith, R. K., Brickhouse, N. S., Liedahl, D. A., \& Raymond, J. C. 2001, ApJ, 556, L91

Tian, F., Toon, O. B., Pavlov, A. A., \& De Sterck, H. 2005, ApJ, 621, 1049

Vidal-Madjar, A., Lecavelier des Etangs, A., Désert, J.-M., et al. 2003, Nature, 422, 143

Watson, A. J., Donahue, T. M., \& Walker, J. C. G. 1981, Icarus, 48, 150

Winn, J. N., Holman, M. J., Henry, G. W., et al. 2007, AJ, 133, 1828

Yelle, R. V. 2004, Icarus, 170, 167 
J. Sanz-Forcada et al.: Estimation of the XUV radiation onto close planets and their evaporation

Table 3. X-ray flux (0.12-2.48 keV) and fits of stars with exoplanets (XMM-Newton and Chandra data).

\begin{tabular}{|c|c|c|c|c|c|}
\hline Star name & $\begin{array}{c}\log f_{X} \\
\left(\operatorname{erg~s}^{-1} \mathrm{~cm}^{-2}\right) \\
\end{array}$ & $\begin{array}{c}\log T \\
(\mathrm{~K})\end{array}$ & $\begin{array}{r}\log \mathrm{EM} \\
\left(\mathrm{cm}^{-3}\right)\end{array}$ & Elements (X) & $\begin{array}{c}{[\mathrm{X} / \mathrm{H}]} \\
\text { (Solar values of Anders \& Grevesse 1989) }\end{array}$ \\
\hline $14 \mathrm{Her}$ & -13.67 & $6.49_{-0.06}^{+0.06}$ & $49.58_{-0.12}^{+0.10}$ & $\mathrm{Fe}$ & $-0.22_{-0.00}^{+0.00}$ \\
\hline 16 Cyg B & $<-14.04$ & 6.30 & $49.40_{-0.57}^{+0.24}$ & $\mathrm{Fe}$ & -0.20 \\
\hline $2 \mathrm{M} 1207 \mathrm{~A}$ & $<-15.27$ & 6.30 & $48.52_{-0.00}^{+0.34}$ & $\mathrm{Fe}$ & -0.20 \\
\hline 30 Ari B & -11.74 & $6.66_{-0.01}^{+0.01}, 6.30_{-0.06}^{+0.08}, 6.91_{-0.00}^{+0.01}$ & $52.02_{-0.03}^{+0.03}, 51.05_{-0.13}^{+0.17}, 51.61_{-0.03}^{+0.03}$ & $\mathrm{Fe}, \mathrm{O}, \mathrm{Ne}$ & $-0.01_{-0.02}^{+0.02},-0.48_{-0.04}^{+0.04},-0.17_{-0.07}^{+0.06}$ \\
\hline $47 \mathrm{UMa}$ & -14.92 & $6.20_{-0.20}^{+0.16}$ & $48.23_{-0.45}^{+0.24}$ & $\mathrm{Fe}$ & $-0.10_{-0.20}^{+0.00}$ \\
\hline $51 \mathrm{Peg}$ & $<-13.93$ & 6.30 & $49.22_{-0.59}^{+0.24}$ & $\mathrm{Fe}$ & -0.20 \\
\hline $55 \mathrm{Cnc}$ & -13.62 & $6.65_{-0.09}^{+0.05}$ & $49.35_{-0.11}^{+0.06}$ & $\mathrm{Fe}$ & $-0.30_{-0.00}^{+0.20}$ \\
\hline$\beta$ Pic & -15.01 & $6.29_{-0.06}^{+0.10}$ & $48.35_{-0.19}^{+0.11}$ & $\mathrm{Fe}$ & $-0.30_{-0.00}^{+0.20}$ \\
\hline$\epsilon$ Eri & -10.89 & $6.60_{-0.01}^{+0.01}, 6.27_{-0.06}^{+0.05}, 6.87_{-0.02}^{+0.02}$ & $50.67_{-0.08}^{+0.05}, 50.29_{-0.09}^{+0.07}, 50.22_{-0.08}^{+0.07}$ & $\mathrm{Fe}, \mathrm{O}, \mathrm{N}, \mathrm{Ne}, \mathrm{C}$ & $-0.17_{-0.02}^{+0.03},-0.25_{-0.05}^{+0.07}, 0.30_{-0.07}^{+0.09},-0.14_{-0.04}^{+0.05}, 0.25_{-0.15}^{+0.17}$ \\
\hline GJ 317 & -13.86 & $6.62_{-0.09}^{+0.09}$ & $48.81_{-0.17}^{+0.12}$ & $\mathrm{Fe}$ & $-0.23_{-0.44}^{+0.46}$ \\
\hline GJ 436 & -14.13 & $6.76_{-0.13}^{+0.15}, 6.26_{-0.20}^{+0.19}$ & $48.59_{-0.32}^{+0.17}, 48.22_{-0.55}^{+0.22}$ & $\mathrm{Fe}$ & $-0.74_{-1.69}^{+0.45}$ \\
\hline GJ 674 & -11.67 & $6.88_{-0.05}^{+0.08}, 6.48_{-0.01}^{+0.01}, 7.13_{-0.02}^{+0.05}$ & $49.62_{-0.15}^{+0.12}, 50.44_{-0.03}^{+0.03}, 49.71_{-0.14}^{+0.04}$ & $\mathrm{Fe}, \mathrm{O}, \mathrm{Ne}$ & $-0.27_{-0.05}^{+0.05},-0.51_{-0.03}^{+0.03},-0.13_{-0.07}^{+0.07}$ \\
\hline GJ 86 & $<-12.74$ & $6.54_{-0.02}^{+0.02}$ & $50.06_{-0.03}^{+0.03}$ & $\mathrm{Fe}$ & $-0.05_{-0.11}^{+0.11}$ \\
\hline GJ 876 & -13.26 & $6.65_{-0.02}^{+0.02}, 6.18_{-0.00}^{+0.00}$ & $48.84_{-0.03}^{+0.02}, 47.93_{-0.33}^{+0.18}$ & $\mathrm{Fe}$ & $-0.37_{-0.07}^{+0.06}$ \\
\hline GQ Lup & -12.92 & $6.89_{-0.07}^{+0.06}, 7.60_{-0.07}^{+0.06}$ & $52.43_{-0.24}^{+0.06}, 52.71_{-0.06}^{+0.04}$ & $\mathrm{Fe}$ & $-0.30_{-0.00}^{+0.09}$ \\
\hline HD 4308 & $<-14.82$ & 6.30 & $48.64_{-1.12}^{+0.28}$ & $\mathrm{Fe}$ & -0.20 \\
\hline HD 20367 & -11.65 & $6.30_{-0.11}^{+0.99}, 6.65_{-0.21}^{+0.12}, 6.95_{-0.02}^{+0.03}$ & $50.71_{-0.44}^{+0.98}, 51.79_{-0.60}^{+0.05}, 51.39_{-0.07}^{+0.05}$ & $\mathrm{Fe}, \mathrm{O}, \mathrm{N}, \mathrm{Ne}$ & $-0.01_{-0.05}^{+0.04},-0.36_{-0.07}^{+0.10}, 0.19_{-0.20}^{+0.18},-0.47_{-0.40}^{+0.21}$ \\
\hline HD 27442 & $<-14.46$ & 6.30 & $48.83_{-0.61}^{+0.24}$ & $\mathrm{Fe}$ & -0.20 \\
\hline HD 46375 & -13.96 & $6.63_{-0.10}^{+0.12}$ & $49.87_{-0.17}^{+0.09}$ & $\mathrm{Fe}$ & $-0.30_{-0.00}^{+0.00}$ \\
\hline HD 49674 & -13.89 & $6.49_{-0.15}^{+0.18}, 6.00_{-0.00}^{+0.00}$ & $50.13_{-0.97}^{+0.11}, 50.83_{-0.00}^{+0.00}$ & $\mathrm{Fe}, \mathrm{O}$ & $0.41_{-0.55}^{+0.37},-0.41_{-0.86}^{+0.19}$ \\
\hline HD 50554 & $<-14.74$ & 6.30 & $49.02_{-0.46}^{+0.22}$ & $\mathrm{Fe}$ & -0.20 \\
\hline HD 52265 & -14.08 & $6.43_{-0.07}^{+0.12}$ & $49.46_{-0.33}^{+0.23}$ & $\mathrm{Fe}$ & $0.27_{-N / A}^{+0.51}$ \\
\hline HD 70642 & -14.61 & $6.53_{-0.12}^{+0.21}$ & $49.08_{-0.29}^{+0.16}$ & $\mathrm{Fe}$ & $-0.30_{-0.00}^{+0.20}$ \\
\hline HD 75289 & $<-15.10$ & 6.30 & $48.60_{-N / A}^{+0.34}$ & $\mathrm{Fe}$ & -0.20 \\
\hline HD 93083 & -14.10 & $6.77_{-0.22}^{+0.17}$ & $49.74_{-0.26}^{+0.15}$ & $\mathrm{Fe}$ & $-1.12_{-N / A}^{+0.98}$ \\
\hline HD 95089 & $<-15.18$ & 6.30 & $47.94_{-N / A}^{+0.31}$ & $\mathrm{Fe}$ & -0.20 \\
\hline HD 99492 & -14.04 & $6.59_{-0.07}^{+0.10}$ & $49.23_{-0.15}^{+0.12}$ & $\mathrm{Fe}$ & $-0.22_{-0.59}^{+0.42}$ \\
\hline HD 101930 & -14.99 & 6.30 & $48.75_{-N / A}^{+0.41}$ & $\mathrm{Fe}$ & -0.20 \\
\hline HD 102195 & -12.57 & $6.74_{-0.02}^{+0.02}, 6.01_{-0.01}^{+0.04}$ & $50.80_{-0.05}^{+0.04}, 51.04_{-0.14}^{+0.08}$ & $\mathrm{Fe}$ & $-0.05_{-0.06}^{+0.07}$ \\
\hline HD 108147 & -13.86 & $6.60_{-0.13}^{+0.16}$ & $50.05_{-0.26}^{+0.15}$ & $\mathrm{Fe}$ & $-0.10_{-0.00}^{+0.00}$ \\
\hline HD 111232 & $<-14.65$ & 6.30 & $49.05_{-0.00}^{+0.06}$ & $\mathrm{Fe}$ & -0.20 \\
\hline HD 114386 & -14.44 & $6.39_{-0.22}^{+0.20}$ & $49.20_{-0.39}^{+0.20}$ & $\mathrm{Fe}$ & $-0.30_{-0.00}^{+0.20}$ \\
\hline HD 114762 & $<-14.78$ & 6.30 & $49.19_{-N / A}^{+0.43}$ & $\mathrm{Fe}$ & -0.20 \\
\hline HD 114783 & $<-14.16$ & $6.38_{-0.09}^{+0.12}$ & $49.21_{-0.33}^{+0.17}$ & $\mathrm{Fe}$ & $-0.30_{-0.00}^{+0.20}$ \\
\hline HD 130322 & -13.78 & $6.56_{-0.08}^{+0.09}$ & $49.94_{-0.14}^{+0.10}$ & $\mathrm{Fe}$ & $-0.24_{-0.06}^{+0.14}$ \\
\hline HD 154345 & -13.47 & $6.42_{-0.05}^{+0.08}$ & $49.67_{-0.14}^{+0.11}$ & $\mathrm{Fe}$ & $0.31_{-0.78}^{+0.40}$ \\
\hline HD 164922 & $<-15.02$ & 6.30 & $48.44_{-N / A}^{+0.41}$ & $\mathrm{Fe}$ & -0.20 \\
\hline HD 179949 & -12.56 & $6.06_{-0.04}^{+0.05}, 6.72_{-0.01}^{+0.01}$ & $50.77_{-0.17}^{+0.15}, 50.74_{-0.04}^{+0.03}$ & $\mathrm{Fe}$ & $0.12_{-0.04}^{+0.04}$ \\
\hline HD 187123 & $<-14.20$ & 6.30 & $49.98_{-0.00}^{+0.01}$ & $\mathrm{Fe}$ & -0.20 \\
\hline HD 189733 & -12.47 & $6.85_{-0.01}^{+0.01}, 6.13_{-0.01}^{+0.01}, 6.68_{-0.05}^{+0.04}$ & $50.66_{-0.05}^{+0.02}, 50.49_{-0.04}^{+0.03}, 50.23_{-0.14}^{+0.04}$ & $\mathrm{Fe}, \mathrm{O}, \mathrm{Ne}$ & $-0.40_{-0.00}^{+0.00},-0.05_{-0.04}^{+0.02},-0.40_{-0.00}^{+0.00}$ \\
\hline HD 190360 & $<-14.13$ & 6.30 & $49.05_{-0.00}^{+0.01}$ & $\mathrm{Fe}$ & -0.20 \\
\hline HD 195019 & $<-14.99$ & 6.30 & $48.91_{-0.74}^{+0.26}$ & $\mathrm{Fe}$ & $0.00_{-0.00}^{+0.00}$ \\
\hline HD 209458 & $<-15.02$ & 6.30 & $49.10_{-0.00}^{+0.06}$ & $\mathrm{Fe}$ & -0.20 \\
\hline HD 216435 & -13.38 & $6.58_{-0.03}^{+0.04}$ & $50.36_{-0.06}^{+0.05}$ & $\mathrm{Fe}$ & $-0.00_{-0.23}^{+0.00}$ \\
\hline HD 216437 & -14.30 & $6.40_{-0.10}^{+0.21}$ & $49.28_{-0.36}^{+0.19}$ & $\mathrm{Fe}$ & $-0.30_{-0.00}^{+0.20}$ \\
\hline HD 217107 & $<-15.17$ & 6.30 & $48.20_{-N / A}^{+0.45}$ & $\mathrm{Fe}$ & -0.20 \\
\hline HD 218566 & -13.99 & $6.52_{-0.16}^{+0.17}$ & $49.72_{-0.39}^{+0.21}$ & $\mathrm{Fe}$ & $-0.24_{-N / A}^{+0.92}$ \\
\hline HD 330075 & -14.97 & $6.60_{-0.24}^{+1.30}$ & $49.22_{-N / A}^{+0.30}$ & $\mathrm{Fe}$ & $-0.30_{-0.00}^{+0.20}$ \\
\hline HR 8799 & -13.26 & $6.54_{-0.08}^{+0.07}$ & $50.75_{-0.14}^{+0.11}$ & $\mathrm{Fe}$ & $-0.55_{-N / A}^{+0.60}$ \\
\hline$\mu$ Ara & $<-14.46$ & 6.30 & $48.68_{-0.26}^{+0.16}$ & $\mathrm{Fe}$ & -0.20 \\
\hline NGC 24233 & $<-14.58$ & 6.30 & $56.27_{-N / A}^{+1.58}$ & $\mathrm{Fe}$ & -0.20 \\
\hline Pollux & -12.97 & $6.23_{-0.12}^{+0.05}, 6.54_{-0.43}^{+0.21}$ & $49.90_{-0.11}^{+0.06}, 48.70_{-1.68}^{+1.08}$ & $\mathrm{Fe}, \mathrm{O}$ & $0.43_{-0.34}^{+0.35},-0.36_{-0.15}^{+0.30}$ \\
\hline$\tau$ Boo & -11.51 & $6.62_{-0.01}^{+0.01}, 6.30_{-0.04}^{+0.04}, 6.92_{-0.01}^{+0.01}$ & $51.70_{-0.02}^{+0.02}, 50.94_{-0.05}^{+0.08}, 50.93_{-0.04}^{+0.06}$ & $\begin{array}{c}\mathrm{Fe}, \mathrm{O}, \mathrm{Ne}, \mathrm{Mg} \\
\mathrm{Si}, \mathrm{C}, \mathrm{N}\end{array}$ & $\begin{array}{c}-0.28_{-0.00}^{+0.00},-0.56_{-0.02}^{+0.03},-0.51_{-0.06}^{+0.05},-0.28_{-0.03}^{+0.05} \\
\quad-0.09_{-0.04}^{+0.05},-0.22_{-0.00}^{+0.00},-0.37_{-0.00}^{+0.00}\end{array}$ \\
\hline$v$ And & -12.77 & $6.53_{-0.02}^{+0.02}$ & $50.09_{-0.04}^{+0.03}$ & $\mathrm{Fe}$ & $0.30_{-0.09}^{+0.09}$ \\
\hline
\end{tabular}


Table 4. Stars with exoplanets. XUV luminosity predicted in different bands ${ }^{a}$.

\begin{tabular}{|c|c|c|c|c|c|c|}
\hline \multirow[t]{2}{*}{ Star } & \multicolumn{6}{|c|}{ Luminosity ( $\mathrm{erg} \mathrm{s}^{-1}$ ) in the wavelength ranges indicated (in $\AA$ ) } \\
\hline & $100-200$ & $200-300$ & $300-400$ & $400-550$ & $550-700$ & $700-920$ \\
\hline $14 \mathrm{Her}$ & $26.01 \pm 0.08$ & $26.61 \pm 0.15$ & $27.10 \pm 0.39$ & $26.63 \pm 0.39$ & $27.06 \pm 0.44$ & $27.34 \pm 0.48$ \\
\hline 16 Cyg B & $<26.32$ & $<27.00$ & $<27.37$ & $<26.89$ & $<27.33$ & $<27.65$ \\
\hline $2 \mathrm{M} 1207 \mathrm{~A}$ & $<25.44$ & $<26.12$ & $<26.49$ & $<26.01$ & $<26.45$ & $<26.77$ \\
\hline 30 Ari B & $28.65 \pm 0.00$ & $28.90 \pm 0.00$ & $28.83 \pm 0.05$ & $28.19 \pm 0.05$ & $27.92 \pm 0.20$ & $28.02 \pm 0.35$ \\
\hline $47 \mathrm{UMa}$ & $25.64 \pm 0.01$ & $25.88 \pm 0.04$ & $25.86 \pm 0.31$ & $25.29 \pm 0.38$ & $25.74 \pm 0.42$ & $26.00 \pm 0.48$ \\
\hline $51 \mathrm{Peg}$ & $<26.14$ & $<26.82$ & $<27.19$ & $<26.71$ & $<27.15$ & $<27.47$ \\
\hline $55 \mathrm{Cnc}$ & $25.68 \pm 0.09$ & $26.10 \pm 0.28$ & $26.84 \pm 0.43$ & $26.37 \pm 0.42$ & $26.83 \pm 0.44$ & $27.11 \pm 0.49$ \\
\hline$\beta$ Pic & $25.31 \pm 0.07$ & $25.91 \pm 0.14$ & $26.34 \pm 0.43$ & $25.87 \pm 0.42$ & $26.33 \pm 0.44$ & $26.61 \pm 0.49$ \\
\hline$\epsilon$ Eri & $27.69 \pm 0.00$ & $27.96 \pm 0.00$ & $27.81 \pm 0.00$ & $27.26 \pm 0.00$ & $27.31 \pm 0.00$ & $27.49 \pm 0.00$ \\
\hline GJ 86 & $<26.85$ & $<27.64$ & $<28.50$ & $<28.02$ & $<28.49$ & $<28.81$ \\
\hline GJ 317 & $25.32 \pm 0.19$ & $25.95 \pm 0.36$ & $26.77 \pm 0.45$ & $26.31 \pm 0.45$ & $26.79 \pm 0.44$ & $27.07 \pm 0.49$ \\
\hline GJ 436 & $25.26 \pm 0.05$ & $25.66 \pm 0.19$ & $26.21 \pm 0.42$ & $25.75 \pm 0.41$ & $26.21 \pm 0.44$ & $26.48 \pm 0.48$ \\
\hline GJ 674 & $26.94 \pm 0.01$ & $27.37 \pm 0.03$ & $27.49 \pm 0.24$ & $26.97 \pm 0.20$ & $27.02 \pm 0.37$ & $27.29 \pm 0.46$ \\
\hline GJ 876 & $25.44 \pm 0.02$ & $25.65 \pm 0.10$ & $26.01 \pm 0.34$ & $25.52 \pm 0.35$ & $25.93 \pm 0.42$ & $26.20 \pm 0.48$ \\
\hline GQ Lup & $29.17 \pm 0.11$ & $29.51 \pm 0.41$ & $30.38 \pm 0.47$ & $29.92 \pm 0.45$ & $30.41 \pm 0.44$ & $30.69 \pm 0.49$ \\
\hline HD 4308 & $<25.56$ & $<26.24$ & $<26.61$ & $<26.13$ & $<26.57$ & $<26.89$ \\
\hline HD 20367 & $28.44 \pm 0.01$ & $28.67 \pm 0.05$ & $28.90 \pm 0.26$ & $28.25 \pm 0.26$ & $28.51 \pm 0.42$ & 0.48 \\
\hline HD 27442 & $<25.75$ & $<26.43$ & $<26.80$ & $<26.32$ & $<26.76$ & $<27.08$ \\
\hline HD 46375 & $26.37 \pm 0.18$ & $27.00 \pm 0.36$ & $27.83 \pm 0.46$ & $27.37 \pm 0.45$ & $27.85 \pm 0.44$ & 28.13 \\
\hline HD 49674 & $29.06 \pm 0.00$ & $28.43 \pm 0.04$ & $28.61 \pm 0.21$ & $27.88 \pm 0.32$ & $28.11 \pm 0.38$ & $28.41 \pm 0.44$ \\
\hline HD 50554 & $<25.94$ & $<26.62$ & $<26.99$ & $<26.51$ & $<26.95$ & $<27.27$ \\
\hline HD 52265 & $26.05 \pm 0.08$ & $26.97 \pm 0.05$ & $27.11 \pm 0.29$ & $26.54 \pm 0.36$ & $26.95 \pm 0.44$ & $27.22 \pm 0.48$ \\
\hline HD 70642 & $25.62 \pm 0.17$ & $26.28 \pm 0.31$ & $27.05 \pm 0.45$ & $26.59 \pm 0.44$ & $27.06 \pm 0.44$ & $27.34 \pm 0.49$ \\
\hline HD 75289 & $<25.52$ & $<26.20$ & $<26.57$ & $<26.09$ & $<26.53$ & $<26.85$ \\
\hline HD 93083 & $26.14 \pm 0.19$ & $26.81 \pm 0.42$ & $27.69 \pm 0.47$ & $27.23 \pm 0.45$ & $27.72 \pm 0.44$ & $28.00 \pm 0.49$ \\
\hline HD 95089 & $<26.71$ & $<27.39$ & $<27.76$ & $<27.28$ & $<27.72$ & $<28.04$ \\
\hline HD 99492 & $25.75 \pm 0.18$ & $26.39 \pm 0.34$ & $27.20 \pm 0.45$ & $26.73 \pm 0.44$ & $27.21 \pm 0.44$ & $27.49 \pm 0.49$ \\
\hline HD 101930 & $25.63 \pm 0.03$ & $26.27 \pm 0.05$ & $26.32 \pm 0.35$ & $25.84 \pm 0.36$ & $26.24 \pm 0.43$ & $26.52 \pm 0.48$ \\
\hline HD 102195 & $28.84 \pm 0.01$ & $28.51 \pm 0.17$ & $29.09 \pm 0.36$ & $28.57 \pm 0.41$ & $29.03 \pm 0.43$ & $29.31 \pm 0.48$ \\
\hline HD 108147 & $26.59 \pm 0.19$ & $27.21 \pm 0.34$ & $28.02 \pm 0.45$ & $27.55 \pm 0.44$ & $28.03 \pm 0.44$ & $28.31 \pm 0.49$ \\
\hline HD 111232 & $<25.97$ & $<26.65$ & $<27.02$ & $<26.54$ & $<26.98$ & $<27.30$ \\
\hline HD 114386 & $25.79 \pm 0.05$ & $26.45 \pm 0.10$ & $26.73 \pm 0.38$ & $26.27 \pm 0.37$ & $26.69 \pm 0.44$ & $26.96 \pm 0.48$ \\
\hline HD 114762 & $<26.11$ & $<26.79$ & $<27.16$ & $<26.68$ & $<27.12$ & $<27.44$ \\
\hline HD 114783 & $<25.90$ & $<26.62$ & $<27.17$ & $<26.69$ & $<27.14$ & $<27.46$ \\
\hline HD 130322 & $26.47 \pm 0.18$ & $27.13 \pm 0.32$ & $27.91 \pm 0.45$ & $27.44 \pm 0.44$ & $27.92 \pm 0.44$ & $28.20 \pm 0.49$ \\
\hline HD 154345 & $26.29 \pm 0.08$ & $27.24 \pm 0.05$ & $27.34 \pm 0.28$ & $26.76 \pm 0.36$ & $27.16 \pm 0.44$ & $27.43 \pm 0.48$ \\
\hline HD 164922 & $<25.36$ & $<26.04$ & $<26.41$ & $<25.93$ & $<26.37$ & $<26.69$ \\
\hline HD 179949 & $28.70 \pm 0.01$ & $28.42 \pm 0.12$ & $28.83 \pm 0.36$ & $28.29 \pm 0.42$ & $28.76 \pm 0.43$ & $29.04 \pm 0.48$ \\
\hline HD 187123 & $<26.90$ & $<27.58$ & $<27.95$ & $<27.47$ & $<27.91$ & $<28.23$ \\
\hline HD 189733 & $27.90 \pm 0.00$ & $27.86 \pm 0.02$ & $27.84 \pm 0.13$ & $27.08 \pm 0.19$ & $27.48 \pm 0.27$ & $27.60 \pm 0.43$ \\
\hline HD 190360 & $<25.97$ & $<26.65$ & $<27.02$ & $<26.54$ & $<26.98$ & $<27.30$ \\
\hline HD 195019 & $<25.92$ & $<26.64$ & $<26.89$ & $<26.40$ & $<26.84$ & $<27.16$ \\
\hline HD 209458 & $<26.02$ & $<26.70$ & $<27.07$ & $<26.59$ & $<27.03$ & $<27.35$ \\
\hline HD 216435 & $26.92 \pm 0.19$ & $27.56 \pm 0.31$ & $28.34 \pm 0.44$ & $27.86 \pm 0.44$ & $28.34 \pm 0.44$ & $28.62 \pm 0.49$ \\
\hline HD 216437 & $25.83 \pm 0.06$ & $26.50 \pm 0.10$ & $26.81 \pm 0.38$ & $26.35 \pm 0.37$ & $26.77 \pm 0.43$ & $27.04 \pm 0.48$ \\
\hline HD 217107 & $<25.12$ & $<25.80$ & $<26.17$ & $<25.69$ & $<26.13$ & $<26.45$ \\
\hline HD 218566 & $26.27 \pm 0.17$ & $26.94 \pm 0.29$ & $27.69 \pm 0.45$ & $27.23 \pm 0.44$ & $27.70 \pm 0.44$ & $27.98 \pm 0.49$ \\
\hline HD 330075 & $25.56 \pm 0.09$ & $26.02 \pm 0.25$ & $26.71 \pm 0.42$ & $26.25 \pm 0.41$ & $26.70 \pm 0.44$ & $26.98 \pm 0.49$ \\
\hline HR 8799 & $27.26 \pm 0.16$ & $27.92 \pm 0.33$ & $28.71 \pm 0.46$ & $28.26 \pm 0.44$ & $28.73 \pm 0.44$ & $29.01 \pm 0.49$ \\
\hline & & $<2628$ & & $<26.17$ & $<26.61$ & $<26.93$ \\
\hline NGC 24233 & $<33.19$ & $<33.87$ & $<34.24$ & $<33.76$ & $<34.20$ & $<34.52$ \\
\hline Pollux & $27.68 \pm 0.01$ & $27.98 \pm 0.01$ & $27.66 \pm 0.23$ & $26.93 \pm 0.34$ & $27.18 \pm 0.41$ & $27.47 \pm 0.48$ \\
\hline$\tau$ Boo & $28.21 \pm 0.01$ & $28.47 \pm 0.12$ & $28.97 \pm 0.37$ & $28.35 \pm 0.27$ & $28.50 \pm 0.42$ & $28.77 \pm 0.49$ \\
\hline$v$ And & $26.75 \pm 0.20$ & $27.46 \pm 0.22$ & $28.10 \pm 0.40$ & $27.60 \pm 0.44$ & $28.07 \pm 0.44$ & $28.35 \pm 0.49$ \\
\hline
\end{tabular}

Notes. ${ }^{(a)}$ Only stars with XMM-Newton or Chandra data. 


\section{Appendix A: Extrapolation of the lower temperature EMD}

The determination of the EMD in the transition region ( $\log T[\mathrm{~K}] \sim 4.2-5.8)$ usually benefits from the information provided by UV lines. For the sources without UV spectroscopic observations we need to develop a method to calculate the EMD in this region. We extrapolate the values of the EMD at those temperatures based on the coronal counterpart, for which a general proportionality seems to be present. Both transition region and coronal material are supposed to be part of the same geometrical structures (loops). In the coronal EMD of all sources we can identify material at $\log T[\mathrm{~K}] \sim 6.3$, the typical temperature of the solar corona, despite of their activity level. We use the EM level at that peak, averaged over three values of $T$, to calibrate the relation to the lower temperature EMD. We used a sample of objects with a well calculated EMD over the whole range, using same technique in all cases (Sanz-Forcada et al. 2002; Sanz-Forcada \& Micela 2002; Sanz-Forcada et al. 2003a; Huenemoerder et al. 2003; Sanz-Forcada et al. 2004), and adding $\alpha$ Cen B (Sect. B). We separated the sample in three groups according to the level of activity (interpreted from the amount of EMD found at the highest temperatures): low activity stars (group 1: Procyon, $\alpha$ Cen B), moderately active stars (group 2: $\epsilon$ Eri, $\xi$ UMa B), and active stars (group 3: VY Ari, $\sigma^{2} \mathrm{CrB}$, AR Lac, FK Aqr, AD Leo, UX Ari, V711 Tau, II Peg, AB Dor).

The lower temperature EMD can be defined using three parameters (see Fig. A.1, Table A.1). Two come from the fitting of the EMD with a straight line: the slope of this line and the difference between the minimum EM (at $T_{\min }$ ) and the local maximum at $\log T[\mathrm{~K}]=6.2-6.4\left(\Delta \mathrm{EM}_{1}\right)$. The fit makes use of values in the temperature range $\log T[\mathrm{~K}]=4.2-T_{\min }$. Since groups 1 and 2 have only four objects between them, we applied the same model to all of them.

We also need a way to account for the different sampling of the EMD in $T$, from the 0.1 dex binning used in the EMD to the 3-temperature fit typical in low-resolution spectra. The fits with one or two temperatures are assumed to be like the 3-T fits with the remaining temperatures considered as negligible. The third parameter needed in our model accounts for this binning in the form of a vertical shift of the EM $\left(\Delta \mathrm{EM}_{2}\right)$ to be added to $\Delta \mathrm{EM}_{1}$. This parameter shows a dependence on the level of activity, according to the distribution of mass in temperature. We used a representative star for each group, all of them of spectral type K2V: $\alpha$ Cen B (group 1), $\epsilon$ Eri (group 2), and AB Dor (group 3). The $\Delta \mathrm{EM}_{2}$ of each case is listed in Table A.1.

Depending on the temperature and EM found in the targets in our sample, we use one of the three groups and extrapolate the EM of the transition region using the value of EM at the temperature closer to $\log T(\mathrm{~K})=6.3\left(\mathrm{EM}_{6.3}\right)$ : we first determine the EM of $T_{\text {min }}$ (using $\log T_{\min }(\mathrm{K})=5.7$ ): $\mathrm{EM}_{\min }=\mathrm{EM}_{\log T \sim 6.3}-$ $\Delta \mathrm{EM}_{1}-\Delta \mathrm{EM}_{2}$. Then we extend the EM at lower temperatures with a straight line with the slope in Table A.1, resulting in the values listed in Table A.2. Uncertainties in the lower temperature EMD are calculated based on those from Table A.1.

We tested the accuracy of the calculation with this method. We used the same three representative stars ( $\alpha$ Cen B, $\epsilon$ Eri, and $\mathrm{AB}$ Dor) with a complete EMD calculated using UV lines and compared this to the flux in same spectral ranges using 3-T model combined with the extrapolated EM at lower temperatures. The values measured from both models (Table A.3) are very similar, so we are confident that the approach followed is correct.

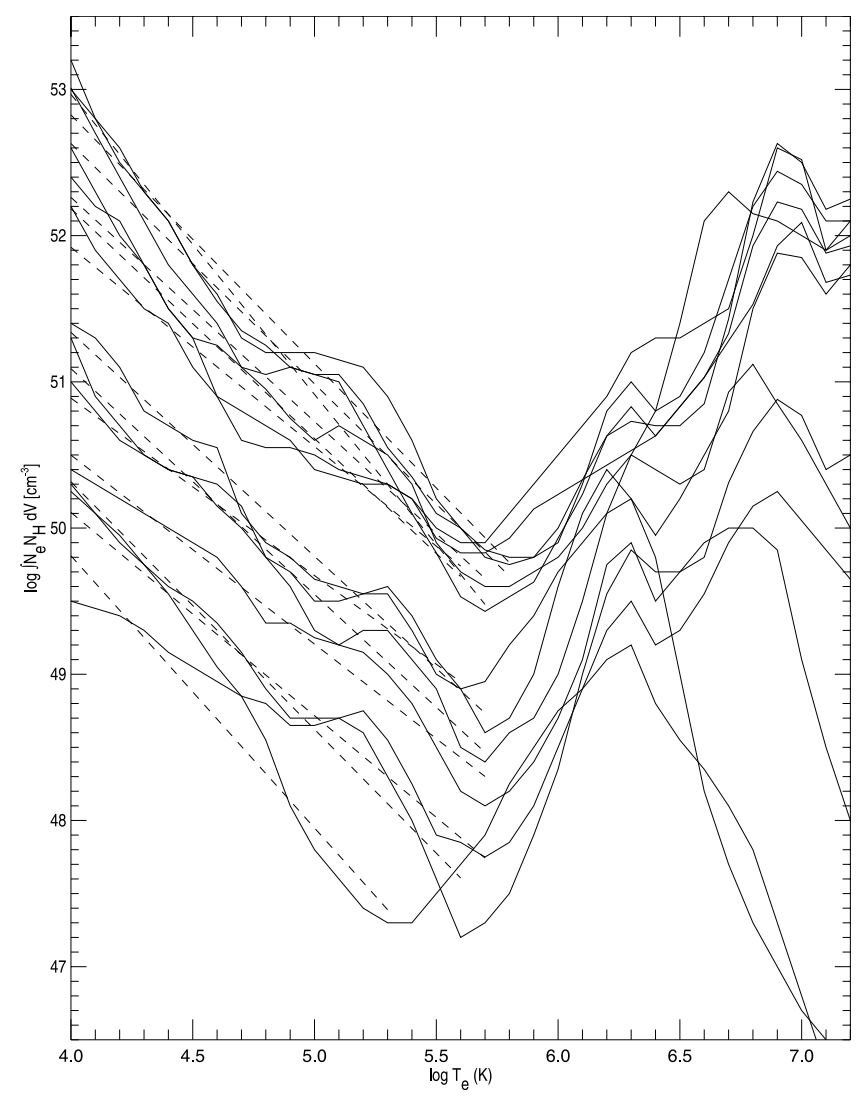

Fig. A.1. Linear fits (dashed lines) applied to the cool side of the EMD (solid lines) of well known coronal models.

Table A.1. Transition region EMD. Fit parameters.

\begin{tabular}{lccc}
\hline \hline Activity level & Slope & $\Delta \mathrm{EM}_{1}\left(\mathrm{~cm}^{-3}\right)$ & $\Delta \mathrm{EM}_{2}\left(\mathrm{~cm}^{-3}\right)$ \\
\hline Low & $-1.66 \pm 0.15$ & $1.84 \pm 0.36$ & 0.6 \\
Medium & $-1.66 \pm 0.15$ & $1.84 \pm 0.36$ & 0.1 \\
High & $-1.53 \pm 0.26$ & $1.19 \pm 0.25$ & 1.8 \\
\hline
\end{tabular}

Finally, we compared the calculation of the EUV flux of $\epsilon$ Eri with the direct EUVE spectrum. The luminosity in the band 80-170 $\AA$ in the observed spectrum was $3.2 \mathrm{e}+27 \mathrm{erg} \mathrm{s}^{-1}$, in the model based on the whole EMD was $2.7 \mathrm{e}+27$, and in the model based on the $3 \mathrm{~T}+$ extrapolated EMs we obtain $1.9 \mathrm{e}+27$. These differences are very small considering the process followed to obtain the synthetic spectra. We are confident that the method can be safely applied to all late-type stars (late F to mid M spectral types). 


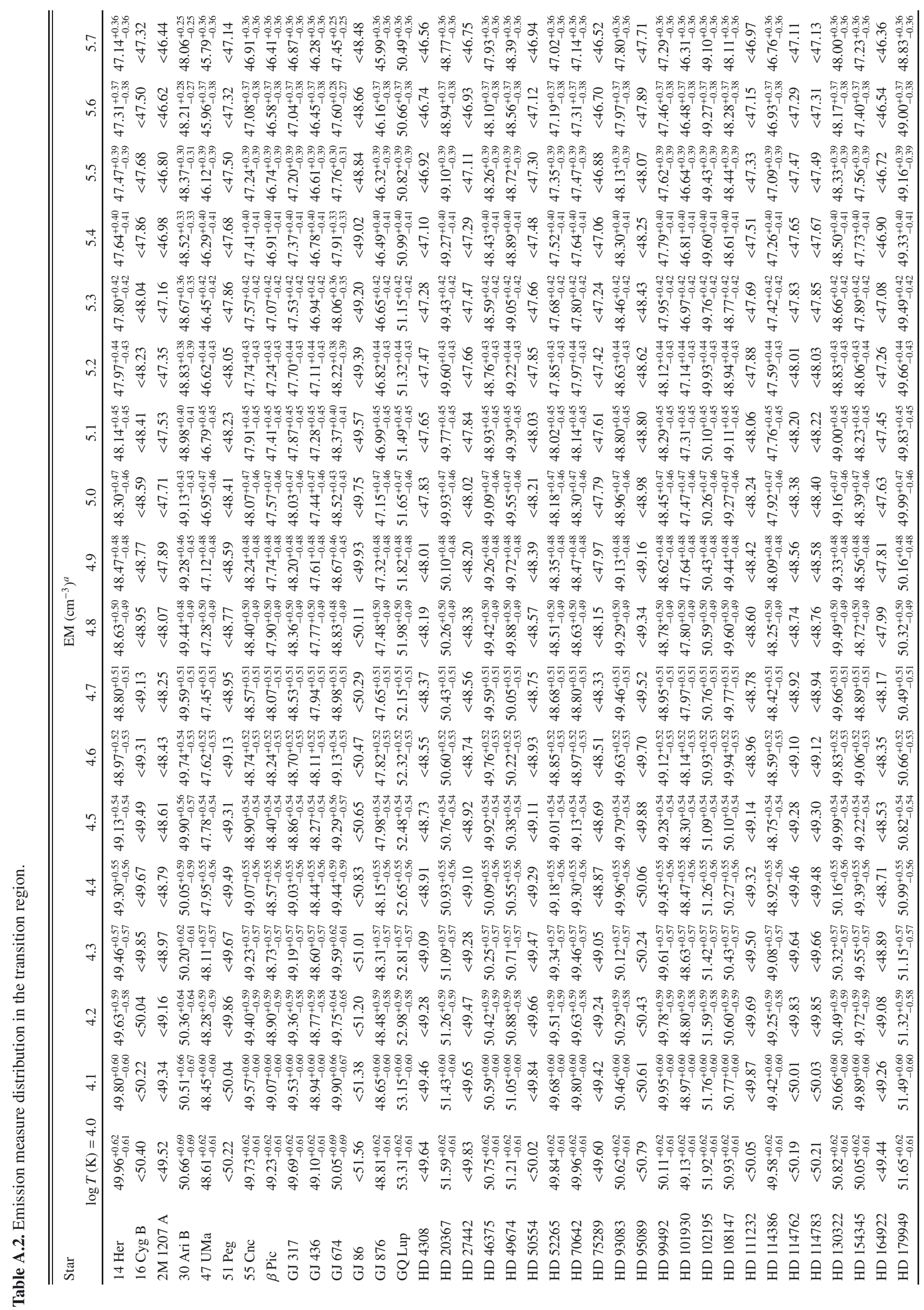




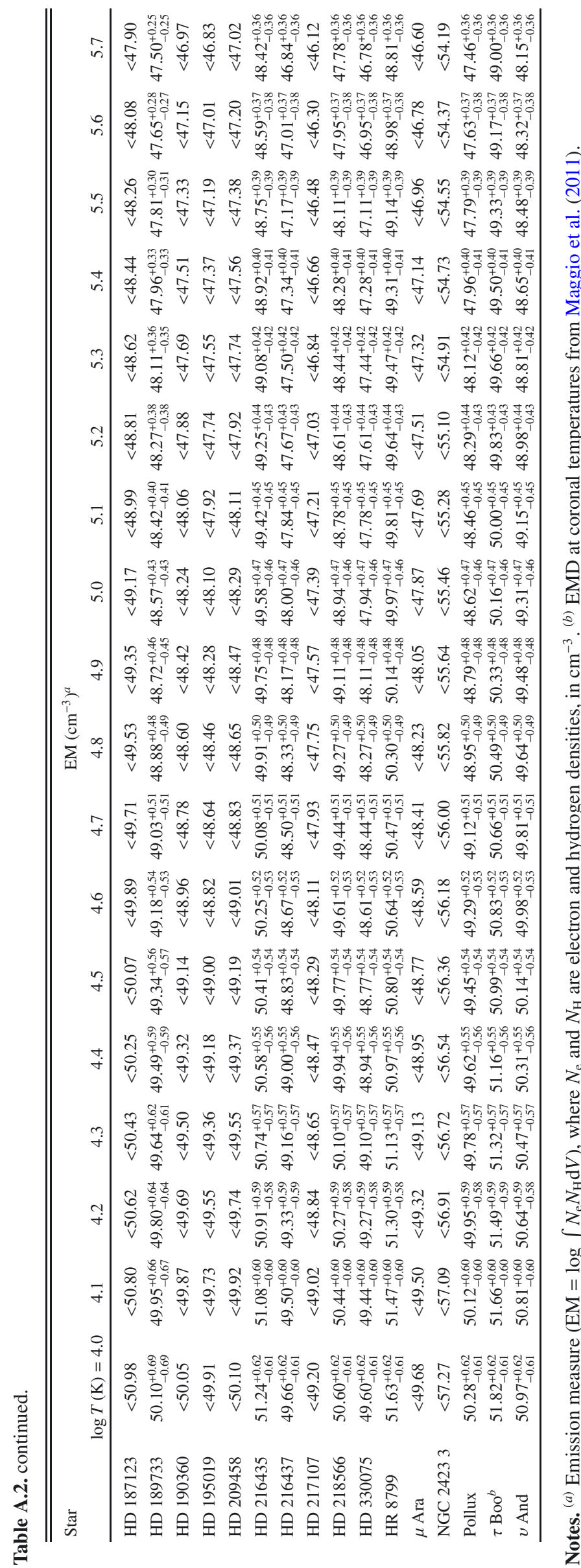

Table A.3. Comparison of fluxes depending on models used ${ }^{a}$.

\begin{tabular}{ccccccc}
\hline \hline & \multicolumn{4}{c}{$L\left(\mathrm{erg} \mathrm{s}^{-1}\right)$} & \multicolumn{2}{c}{ AB Dor } \\
Range & \multicolumn{2}{c}{$\alpha$ Cen B } & \multicolumn{2}{c}{$\epsilon$ Eri } & EMD & $3 \mathrm{~T}$ \\
$(\AA)$ & EMD & 3T & EMD & $3 \mathrm{~T}$ & EM \\
\hline $5-100$ & $7.0 \mathrm{e}+26$ & $9.3 \mathrm{e}+26$ & $1.8 \mathrm{e}+28$ & $1.6 \mathrm{e}+28$ & $2.1 \mathrm{e}+30$ & $1.6 \mathrm{e}+30$ \\
$100-920$ & $6.8 \mathrm{e}+27$ & $4.1 \mathrm{e}+27$ & $2.7 \mathrm{e}+28$ & $1.7 \mathrm{e}+28$ & $6.3 \mathrm{e}+29$ & $4.2 \mathrm{e}+29$ \\
\hline
\end{tabular}

Notes. ${ }^{(a)}$ The "3T" model also includes the predicted EM values in the transition region.

Table B.1. XMM/RGS line fluxes of $\alpha$ Cen $\mathrm{B}^{a}$.

\begin{tabular}{|c|c|c|c|c|c|c|}
\hline Ion & $\lambda_{\text {model }}$ & $\log T_{\max }$ & $F_{\text {obs }}$ & $S / N$ & ratio & Blends \\
\hline Ne IX & 13.4473 & 6.6 & $9.59 \mathrm{e}-15$ & 5.6 & -0.10 & $\begin{array}{l}\text { Fe xix 13.4970, 13.5180, } \\
\text { Ne Ix } 13.5531\end{array}$ \\
\hline Ne Ix & 13.6990 & 6.6 & $1.30 \mathrm{e}-14$ & 5.3 & 0.08 & Ni XIX 13.7790, Fe xVII 13.8250 \\
\hline Fe xvII & 15.0140 & 6.7 & $6.62 \mathrm{e}-14$ & 10.7 & -0.03 & \\
\hline Fe xvII & 15.2610 & 6.7 & $4.29 \mathrm{e}-14$ & 8.1 & 0.20 & $\begin{array}{l}\text { O vIII } 15.1760, \\
\text { Fe xVII } 15.2509,15.2615\end{array}$ \\
\hline O vIII & 16.0055 & 6.5 & $1.23 \mathrm{e}-14$ & 4.0 & -0.02 & $\begin{array}{l}\text { Fe xVII 15.9956, Fe xvIII 16.0040, } \\
\text { O VIII } 16.0067\end{array}$ \\
\hline Fe xvII & 16.7800 & 6.7 & $1.38 \mathrm{e}-14$ & 3.9 & -0.35 & \\
\hline Fe xvII & 17.0510 & 6.7 & $6.35 \mathrm{e}-14$ & 9.8 & -0.04 & Fe xvII 17.0960 \\
\hline O vII & 18.6270 & 6.3 & $1.16 \mathrm{e}-14$ & 3.1 & -0.10 & \\
\hline O vIII & 18.9671 & 6.5 & $7.65 \mathrm{e}-14$ & 10.6 & 0.00 & O VIII 18.9725 \\
\hline O VII & 21.6015 & 6.3 & $1.29 \mathrm{e}-13$ & 20.2 & 0.02 & \\
\hline O vII & 22.0977 & 6.3 & $1.22 \mathrm{e}-13$ & 12.5 & 0.19 & \\
\hline $\mathrm{N}$ VII & 24.7792 & 6.3 & $3.68 \mathrm{e}-14$ & 11.1 & -0.04 & $\mathrm{~N}$ VII 24.7846 \\
\hline CaxI & 25.3520 & 6.3 & $7.10 \mathrm{e}-15$ & 3.0 & 0.28 & $\mathrm{~N}$ VII 25.4030 \\
\hline $\mathrm{C}$ vI & 28.4652 & 6.2 & $1.32 \mathrm{e}-14$ & 3.3 & -0.27 & Arxv $28.3860, \mathrm{C}$ vI 28.4663 \\
\hline $\mathrm{N}$ VI & 28.7870 & 6.2 & $2.54 \mathrm{e}-14$ & 5.0 & -0.08 & \\
\hline $\mathrm{N}$ VI & 29.5347 & 6.1 & $2.69 \mathrm{e}-14$ & 5.5 & 0.13 & \\
\hline Caxi & 30.4710 & 6.3 & $2.51 \mathrm{e}-14$ & 4.7 & -0.14 & S xIv $30.4270,30.4690$ \\
\hline $\mathrm{C}$ VI & 33.7342 & 6.1 & $1.83 \mathrm{e}-13$ & 12.4 & 0.01 & C vi 33.7396 \\
\hline $\mathrm{C} \mathrm{v}$ & 34.9728 & 6.0 & $1.49 \mathrm{e}-14$ & 3.2 & 0.03 & Ar Ix 35.0240 \\
\hline Caxi & 35.2750 & 6.3 & $1.87 \mathrm{e}-14$ & 3.6 & 0.16 & S XII 35.2750 \\
\hline S XIII & 35.6670 & 6.4 & $3.93 \mathrm{e}-14$ & 5.6 & -0.01 & Са Xı $35.6340,35.7370$ \\
\hline
\end{tabular}

Notes. ${ }^{(a)}$ Line fluxes in erg $\mathrm{cm}^{-2} \mathrm{~s}^{-1} \cdot \lambda_{\text {model }}(\AA)$ is the APED model wavelength corresponding to the measured line. $\log T_{\max }$ indicates the maximum temperature $(K)$ of formation of the line (unweighted by the EMD). "Ratio" is the $\log \left(F_{\text {obs }} / F_{\text {pred }}\right)$ of the line. Blends amounting to more than $5 \%$ of the total flux for each line are indicated.

\section{Appendix B: Emission measure distribution of $\alpha$ Cen B}

We calculated the EMD of the K2V star $\alpha$ Cen B, needed to test the extrapolation of the lower EMD temperature and the synthesis of the EUV spectra. We used the UV lines fluxes measured by Sanz-Forcada et al. (2003a) and the XMM-Newton/RGS lines fluxes listed in Table B.1, from an observation taken on Jan. 2009 (Fig. B.1). The coronal model (the EMD) was constructed following Sanz-Forcada et al. (2003b). The resulting EMD (Table B.2) is displayed in Fig. B.2, with coronal abundances as listed in Table B.3. A global fit to the Chandra/LETG spectrum was applied by Raassen et al. (2003), with similar results in the corona. 


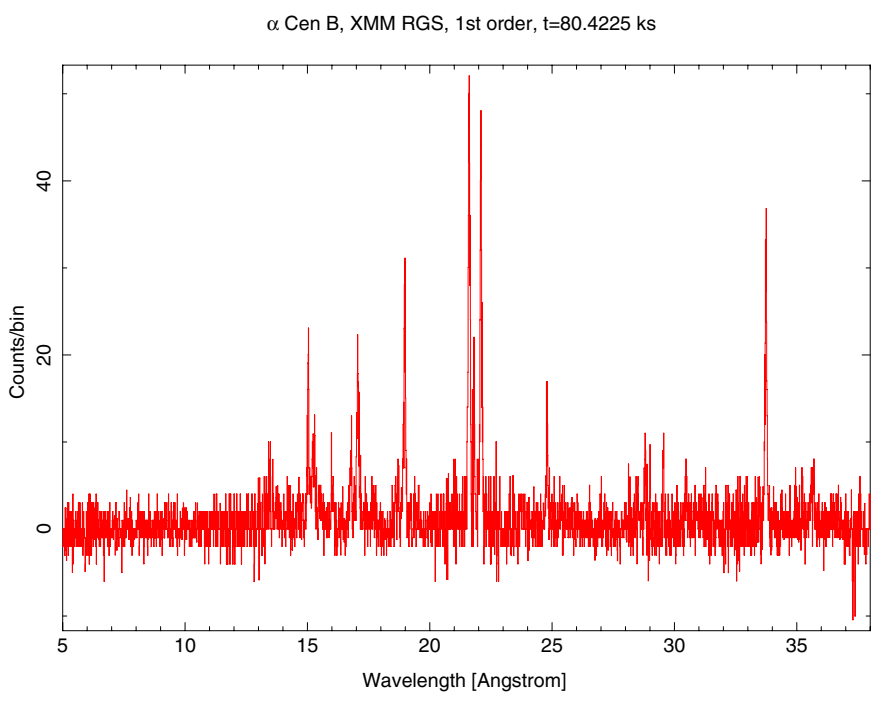

Fig. B.1. XMM-Newton RGS combined spectrum of $\alpha$ Cen B.
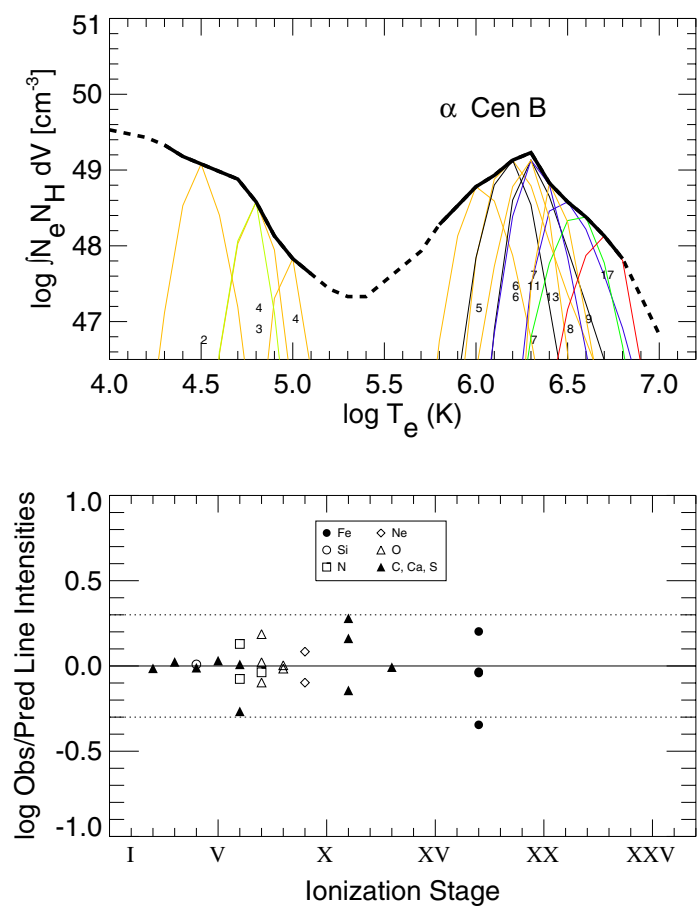

Fig. B.2. Upper panel: EMD of $\alpha$ Cen B. Thin lines represent the relative contribution function for each ion (the emissivity function multiplied by the EMD at each point). Small numbers indicate the ionization stages of the species. Lower panel indicates the observed-to-predicted line flux ratios for the ion stages in the upper figure. The dotted lines denote a factor of 2 .
Table B.2. Emission measure distribution of $\alpha$ Cen B.

\begin{tabular}{cc}
\hline \hline $\log T(\mathrm{~K})$ & $\mathrm{EM}\left(\mathrm{cm}^{-3}\right)^{a}$ \\
\hline 4.0 & $49.53:$ \\
4.1 & $49.48:$ \\
4.2 & $49.43:$ \\
4.3 & $49.33_{-0.30}^{+0.20}$ \\
4.4 & $49.18_{-0.20}^{+0.10}$ \\
4.5 & $49.08_{-0.20}^{+0.10}$ \\
4.6 & $48.98_{-0.20}^{+0.20}$ \\
4.7 & $48.88_{-0.30}^{+0.10}$ \\
4.8 & $48.58_{-0.20}^{+0.20}$ \\
4.9 & $48.13_{-0.10}^{+0.10}$ \\
5.0 & $47.83_{-0.30}^{+0.10}$ \\
5.1 & $47.63_{-0.30}^{+0.20}$ \\
5.2 & $47.43:$ \\
5.3 & $47.33:$ \\
5.4 & $47.33:$ \\
5.5 & $47.53:$ \\
5.6 & $47.73:$ \\
5.7 & $47.93:$ \\
5.8 & $48.28_{-0.40}^{+0.40}$ \\
5.9 & $48.53_{-0.40}^{+0.30}$ \\
6.0 & $48.78_{-0.30}^{+0.20}$ \\
6.1 & $48.93_{-0.40}^{+0.10}$ \\
6.2 & $49.13_{-0.20}^{+0.20}$ \\
6.3 & $49.23_{-0.20}^{+0.10}$ \\
6.4 & $48.83_{-0.20}^{+0.10}$ \\
6.5 & $48.58_{-0.30}^{+0.10}$ \\
6.6 & $48.38_{-0.20}^{+0.20}$ \\
6.7 & $48.13_{-0.40}^{+0.20}$ \\
6.8 & $47.83_{-0.30}^{+0.10}$ \\
6.9 & $47.33:$ \\
7.0 & $46.83:$ \\
\hline & \\
&
\end{tabular}

Notes. ${ }^{(a)}$ Emission measure $\left(\mathrm{EM}=\log \int N_{\mathrm{e}} N_{\mathrm{H}} \mathrm{d} V\right)$, where $N_{\mathrm{e}}$ and $N_{\mathrm{H}}$ are electron and hydrogen densities, in $\mathrm{cm}^{-3}$. Error bars provided are not independent between the different temperatures, as explained in Sanz-Forcada et al. (2003a).

Table B.3. Coronal abundances of $\alpha$ Cen B (solar units ${ }^{a}$ ).

\begin{tabular}{lrccc}
\hline \hline $\mathrm{X}$ & $\mathrm{FIP}(\mathrm{eV})$ & Ref. $^{a}$ & $\left(\mathrm{AG} 89^{a}\right)$ & \multicolumn{1}{c}{$[\mathrm{X} / \mathrm{H}]$} \\
\hline $\mathrm{C}$ & 11.26 & 8.39 & $(8.56)$ & $0.04 \pm 0.11$ \\
$\mathrm{~N}$ & 14.53 & 7.78 & $(8.05)$ & $-0.01 \pm 0.14$ \\
$\mathrm{O}$ & 13.61 & 8.66 & $(8.93)$ & $-0.40 \pm 0.12$ \\
$\mathrm{Ne}$ & 21.56 & 7.84 & $(8.09)$ & $-0.48 \pm 0.19$ \\
$\mathrm{Si}$ & 8.15 & 7.51 & $(7.55)$ & $-0.44 \pm 0.10$ \\
$\mathrm{~S}$ & 10.36 & 7.14 & $(7.21)$ & $0.49 \pm 0.18$ \\
$\mathrm{Ca}$ & 6.11 & 6.31 & $(6.36)$ & $0.22 \pm 0.27$ \\
$\mathrm{Fe}$ & 7.87 & 7.45 & $(7.67)$ & $0.19 \pm 0.19$ \\
\hline
\end{tabular}

Notes. ${ }^{(a)}$ Solar photospheric abundances from Asplund et al. (2005), adopted in this table, are expressed in logarithmic scale. Note that several values have been updated in the literature since Anders \& Grevesse (1989, AG89), also listed in parenthesis for easier comparison. 


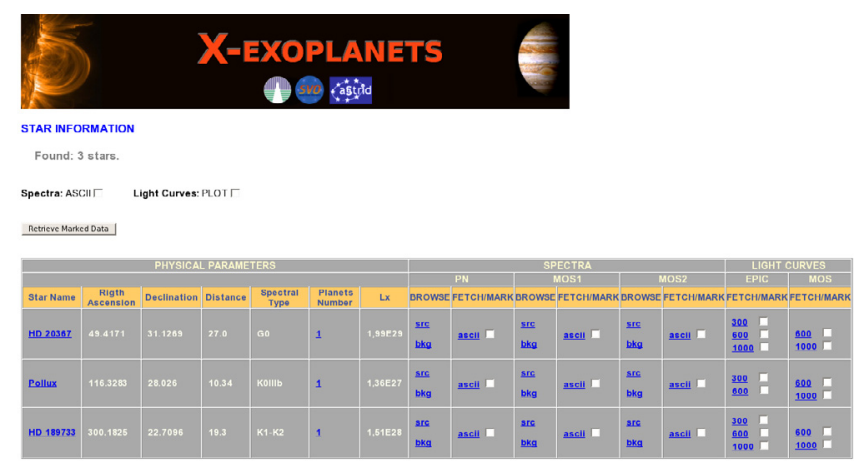

Fig. C.1. The data server X-Exoplanets. Result of a query.

\section{Appendix C: The data server X-Exoplanets}

The data server X-Exoplanets ${ }^{2}$ provides information on the planet-bearing stars that have been observed with XMM-Newton or Chandra. In the near future, synthetic spectra covering the EUV range (Sanz-Forcada et al. 2010a) and EUVE data will also be available. The system contains reduced, science-ready data and was set up to facilitate the analysis of the effects of coronal radiation on exoplanets atmospheres.

\section{C.1. Functionalities: search}

The data server X-Exoplanets is accessed by means of a webbased fill-in form that permits queries by list of objects and coordinates and radius. Searches can be customized to include physical parameters of the stars and planets as well as light curves and reduced spectra obtained from XMM-Newton and Chandra data.

\section{C.2. Functionalities: results}

An example of the result of a query is given in Fig. C.1. Light curves and reduced spectra can be visualized by clicking on the corresponding link (Fig. C.2). The system incorporates multidownload and preview capabilities. Links to SIMBAD and the Extrasolar Planet Encyclopaedia are also provided.

\section{C.3. The Virtual Observatory service}

VO-compliance of an astronomical archive constitutes an added value of enormous importance for the optimum scientific exploitation of their datasets. The X-Exoplanet service has been designed following the IVOA standards and requirements. In particular, it implements the SSA (Simple Spectral Access) protocol and its associated data model, a standard defined for retrieving $1 \mathrm{D}$ data. 
A\&A 532, A6 (2011)
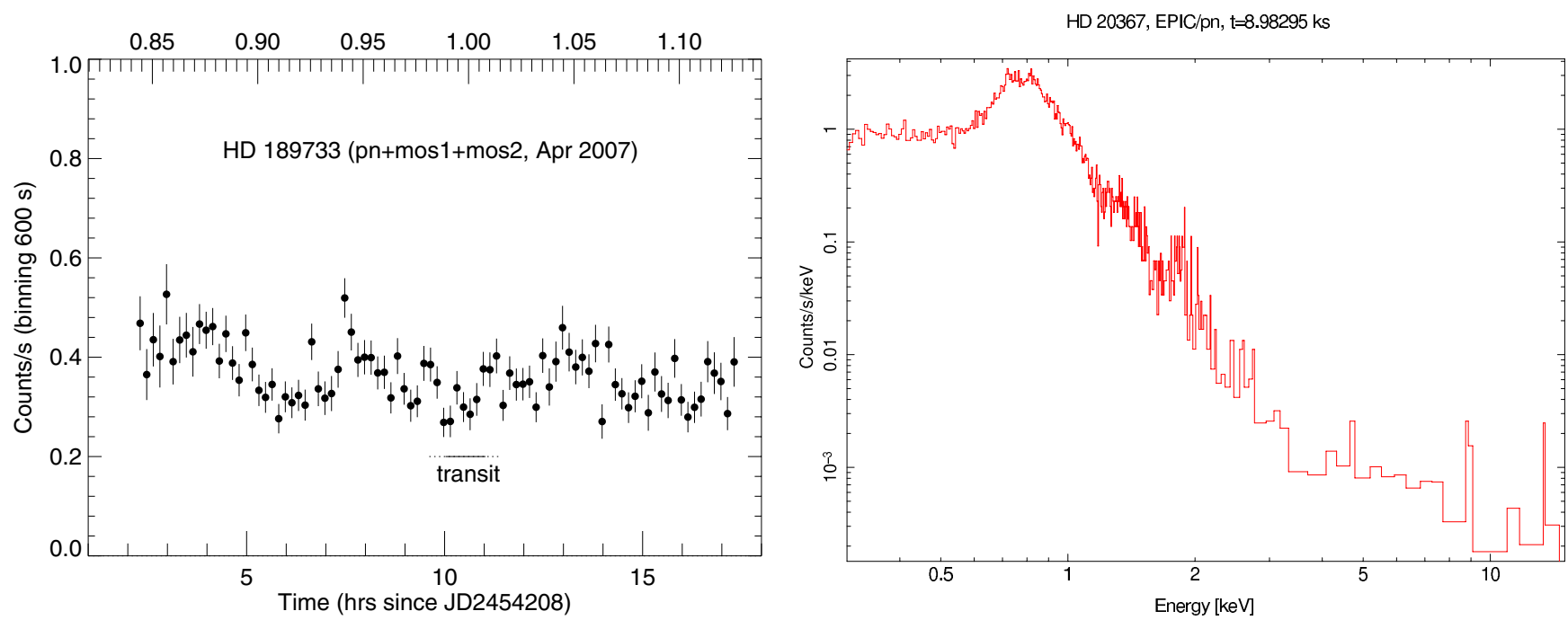

Fig. C.2. The data server X-Exoplanets. Light curve (left) and reduced spectrum (right). In the light curve of HD 189733 we mark the orbital phase (Winn et al. 2007) of HD $189733 \mathrm{~b}$ in the upper axis, as well as the interval when the transit takes place (partial in dotted line, total in solid line). 